\title{
An Efficient Method to Convert Lactams and Amides into 2,2-Dialkylated Amines
}

\author{
Alessandro Agosti, Sebastian Britto ${ }^{\#}$ and Philippe Renaud* \\ Universität Bern, Departement für Chemie und Biochemie, Freiestrasse 3, 3012-Bern, Switzerland \\ ${ }^{\#}$ Research fellow at Universität Bern, on leave from the School of Chemistry, Bharathidasan \\ University, Tiruchirappalli-620 024, India. \\ philippe.renaud@ioc.unibe.ch
}

\section{Supporting Information}

\section{General:}

All reactions were performed under a nitrogen atmosphere in oven-dried flasks $\left(120{ }^{\circ} \mathrm{C}\right)$ unless otherwise stated. Dry solvents for reactions were filtered through a column of dry alumina under a positive pressure of argon. Solvents for flash chromatography were of technical grade and used without purification. Other chemicals were obtained from commercial sources and used without further purifications. The reaction were monitored by TLC (analytical plates, Merck silica gel 60 $\mathrm{F}_{254}$ ) and visualized under UV light and/or stained with a solution of $\mathrm{KMnO}_{4}$ or phosphomolibdic acid followed by heating. Flash chromatography (FC) was performed using Baker silica gel (0.065$0.200 \mathrm{~mm}$ ). Melting points (M.p.) are not corrected. ${ }^{1} \mathrm{H}$ and ${ }^{13} \mathrm{C}$ NMR spectra were recorded on Bruker Avance-300 $\left({ }^{1} \mathrm{H}: 300 \mathrm{MHz},{ }^{13} \mathrm{C}: 75.5 \mathrm{MHz}\right)$ or Bruker DRX-400 $\left({ }^{1} \mathrm{H}: 400 \mathrm{MHz},{ }^{13} \mathrm{C}: 75.5\right.$ $\mathrm{MHz}$ ) spectrometers. The ${ }^{1} \mathrm{H}$ spectra were referred to an internal standard (TMS, $0 \mathrm{ppm}$ ) or to the residual ${ }^{1} \mathrm{H}$ of $\mathrm{CDCl}_{3}(7.26 \mathrm{ppm})$. The ${ }^{13} \mathrm{C}$ spectra were referred to residual signal of $\mathrm{CDCl}_{3}(77.0$ ppm). IR spectra were recorded on Jasco FT-IR 460 plus. Mass spectroscopy (MS) and high resolution mass spectroscopy (HRMS) analysis were performed on Waters Micromass Autospec $Q$ or Qstar Pulsar.

\section{General procedure A: synthesis of thioiminium ions}

Lawesson's reagent $(2.02 \mathrm{~g}, 5.0 \mathrm{mmol})$ was added to a solution of the lactam $(10 \mathrm{mmol})$ in dry $\mathrm{CH}_{2} \mathrm{Cl}_{2}(100 \mathrm{~mL})$. The mixture was stirred at room temperature for $1-4 \mathrm{~h}$ until the starting material was consumed (TLC monitoring). The solvent was evaporated and the crude mixture was filtered on short column of silica gel (cyclohexane/EtOAc). The thiolactam $(10 \mathrm{mmol})$ was suspended in dry THF $(100 \mathrm{ml})$ and MeI $(1.0 \mathrm{~mL}, 16 \mathrm{mmol})$ was added. The mixture was stirred at room 
temperature overnight. The thioiminium ion was isolated by filtration and washed with cold THF to obtain a yellowish solid.

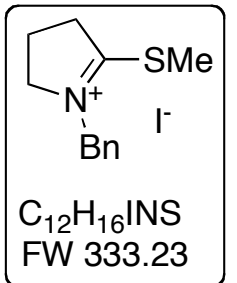

\section{1-Benzyl-5-methylsulfanyl-3,4-didhydro-2H-pyrrolidinium iodide (3a)}

According to general procedure A. $N$-Benzylpyrrolidin-2-one $(1.75 \mathrm{~g}, 10 \mathrm{mmol})$ afforded 3a $(2.73 \mathrm{~g}, 82 \%)$. White solid. M.p. $=94-95{ }^{\circ} \mathrm{C} .{ }^{1} \mathrm{H}$ NMR $\left(\mathrm{CDCl}_{3}, 300\right.$ MHz): $7.42(\mathrm{~m}, 5 \mathrm{H}), 4.94(\mathrm{~s}, 2 \mathrm{H}), 4.19(\mathrm{t}, \mathrm{J}=7.9 \mathrm{~Hz}, 2 \mathrm{H}), 3.78(\mathrm{t}, \mathrm{J}=7.5 \mathrm{~Hz}, 2 \mathrm{H})$, $2.98(\mathrm{~s}, 3 \mathrm{H}), 2.44$ (q, J = 7.9 Hz). ${ }^{13} \mathrm{C} \mathrm{NMR}\left(\mathrm{CDCl}_{3}, 75.5 \mathrm{MHz}\right): 193.54,129.83,129.24,129.15$, 128.87, 59.87, 40.90, 18.98, 17.39. IR (diamond ATR): 1599, 1451, 1444, $1299 \mathrm{~cm}^{-1}$. MS (ESI+): $206\left(100, \mathrm{M}^{+} \mathrm{I}^{+}\right), 176.4$ (14), 102.2 (15). HRMS calcd for $\mathrm{C}_{12} \mathrm{H}_{16} \mathrm{NS}^{+}$206.1003; found 206.1009

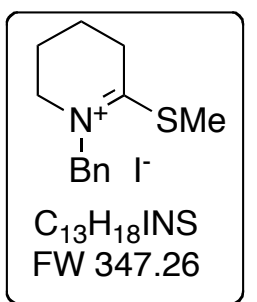

\section{1-Benzyl-6-methylsulfanyl-2,3,4,5-tetrahydropyridinium iodide (3b)}

According to the general procedure A. $N$-Benzylpiperidin-2-one $(1.89 \mathrm{~g}, 10 \mathrm{mmol})$ afforded 3b (2.15g, 62\%). White solid. M.p. $=103-106{ }^{\circ} \mathrm{C} .{ }^{1} \mathrm{H}$ NMR $\left(\mathrm{CDCl}_{3}, 400\right.$ MHz): 7.40 (m, 5H), $5.13(\mathrm{~s}, 2 \mathrm{H}), 3.83(\mathrm{t}, \mathrm{J}=5.5 \mathrm{~Hz}, 2 \mathrm{H}), 3.33(\mathrm{t}, \mathrm{J}=5.9 \mathrm{~Hz}, 2 \mathrm{H})$, 2.92 (s, 3H), 2.14-2.04 (m, 4H). ${ }^{13} \mathrm{C}$ NMR $\left(\mathrm{CDCl}_{3}, 133,3 \mathrm{MHz}\right.$, ): 192.58, 130.13, 129.40, 129.12, 128.24, 60.92, 54.33, 33.32, 20.66, 18.06, 16.82 . IR (diamond ATR): 1597, 1448, $1159 \mathrm{~cm}^{-1}$. MS (ESI $\left.{ }^{+}\right): 220.4\left(100,{\mathrm{M}-I^{+}}^{+}\right), 190.4$ (16). HRMS calcd for $\mathrm{C}_{13} \mathrm{H}_{18} \mathrm{NS}^{+} 220.1159$; found 220.1167.

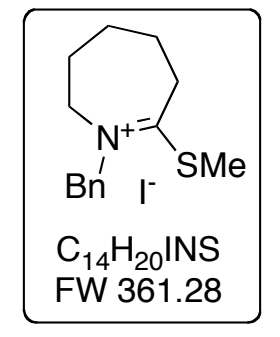

\section{1-Benzyl-7-methylsulfanyl-3,4,5,6-tetrahydro-2H-azepinium iodide (3c)}

According to general procedure starting A. N-Benzylazepan-2-one $(2.033 \mathrm{~g}, 10$ mmol) afforded 3c (2.71 g, 75\%). Yellow solid. Decomposition $124{ }^{\circ} \mathrm{C} .{ }^{1} \mathrm{H}$ NMR $\left(\mathrm{CDCl}_{3}, 400 \mathrm{MHz}\right): 7.36(\mathrm{~m}, 5 \mathrm{H}) 5.17(\mathrm{~s}, 2 \mathrm{H}) 4.17(\mathrm{~m}, 2 \mathrm{H}) 3.44(\mathrm{~m}, 2 \mathrm{H}) 2.94(\mathrm{~s}$, $3 \mathrm{H}) 1.84(\mathrm{~m}, 4 \mathrm{H}) 1.58(\mathrm{~m}, 2 \mathrm{H}) \cdot{ }^{13} \mathrm{C} \mathrm{NMR}\left(\mathrm{CDCl}_{3}, 100 \mathrm{MHz},\right)$ : 197.95, 130.37, 129.50, 128.81, 6.09, 58.56, 34.94, 28.10, 24.95, 24.95, 22.24, 18.92 . IR (diamond ATR): 2922, 1569, $1431 \mathrm{~cm}^{-1}$. MS (ESI $\left.{ }^{+}\right): 234$ (100). HRMS calcd for $\mathrm{C}_{14} \mathrm{H}_{20} \mathrm{NS}^{+}$234.1316; found 234.1324.

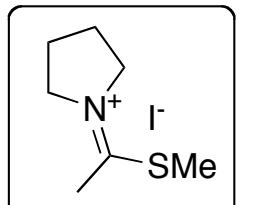

$\mathrm{C}_{7} \mathrm{H}_{14}$ INS FW 271.16

\section{1-(1-(Methylthio)ethylidene)pyrrolidinium iodide (7)}

Prepared according general procedure A. $N$-acetylpyrrolidine $(1,13 \mathrm{~g}, 10.0 \mathrm{mmol})$ afforded 7 (2.62 g, 76\%). Pale yellow solid. Decomposition $115^{\circ} \mathrm{C}$. ${ }^{1} \mathrm{H}$ NMR $\left(\mathrm{CDCl}_{3}, 300 \mathrm{MHz}\right): 4.14(\mathrm{~m}, 2 \mathrm{H}) 3.72(\mathrm{~m}, 2 \mathrm{H}) 2.83(\mathrm{~s}, 3 \mathrm{H}) 2.80(\mathrm{~s}, 3 \mathrm{H}) 2.22(\mathrm{~m}$, $4 \mathrm{H}) .{ }^{13} \mathrm{C} \mathrm{NMR}\left(\mathrm{CDCl}_{3}, 75.5 \mathrm{MHz}\right): 187.52,57.15,55.00,25.17,25.10,25.02$, 
17.81. IR (diamond ATR): 2964, 1598, 1432, $696 \mathrm{~cm}^{-1}$. MS (EI $\left.{ }^{+}\right)$144. HRMS: calcd for $\mathrm{C}_{7} \mathrm{H}_{14} \mathrm{NS}^{+}$ 144.0847; found 144.0850.

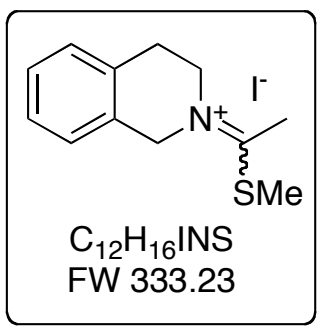

\section{2-(1-(Methylthio)ethylidene)-1,2,3,4-tetrahydroisoquinolinium iodide (13)}

Prepared according general procedure A. $N$-acetyl-1,2,3,4tetrahydroisoquinoline $(1.36 \mathrm{~g}, 7.9 \mathrm{mmol})$ afforded $13(1.17 \mathrm{~g}, 45 \%)$. Orange solid. M.p. $=115-119{ }^{\circ} \mathrm{C} .{ }^{1} \mathrm{H}$ NMR $\left(\mathrm{CDCl}_{3}, 300 \mathrm{MHz}\right): 7.37-7.19(\mathrm{~m}, 8 \mathrm{H})$ $5.27(\mathrm{~s}, 2 \mathrm{H}) 4.94(\mathrm{~s}, 2 \mathrm{H}) 4.27-4.23(\mathrm{t}, \mathrm{J}=3 \mathrm{~Hz}, 2 \mathrm{H}) 3.97-3.93(\mathrm{t}, \mathrm{J}=6 \mathrm{~Hz}, 2 \mathrm{H})$ 3.20-3.18 (t, J =6Hz, 2H) $3.01(\mathrm{~s}, 3 \mathrm{H}) 2.99(\mathrm{~s}, 3 \mathrm{H}) 2.98(\mathrm{~s}, 3 \mathrm{H}) 2.88(\mathrm{~s}, 3 \mathrm{H}) .{ }^{13} \mathrm{C} \mathrm{NMR}\left(\mathrm{CDCl}_{3}\right.$, 75.5 MHz, ): 191.44, 191.12, 134.06, 133.74, 129.48, 128.86, 128.72, 128.32, 127.94, 127.71, $127.62,127.39,127.12,126.58,56.05,54.52,53.02,51.96,28.34,27.84,24.49,18.46,18.39$. IR (diamond ATR): 2967, 1980, 1496, 1243, $608 \mathrm{~cm}^{-1} . \mathrm{MS}\left(\mathrm{EI}^{+}\right)$: 206 (22.6), 132 (22.6). HRMS: calcd. for $\mathrm{C}_{12} \mathrm{H}_{16} \mathrm{NS}^{+}$206.1003; found 206.1000.

\section{General procedure B: bisallylation of thioiminium ions}

Allylmagnesium bromide $(15 \mathrm{~mL}, 15 \mathrm{mmol})$ was added to a suspension of thioiminium ion (5 $\mathrm{mmol})$ in dry THF $(50 \mathrm{~mL})$ at $0^{\circ} \mathrm{C}$, the cooling bath was removed and stirring was continued for 5 h. The mixture was treated with saturated ammonium chloride solution and the aqueous phase extracted with $\mathrm{CH}_{2} \mathrm{Cl}_{2}$. The collected organic phases were washed with brine and dried over $\mathrm{MgSO}_{4}$. The solvent was evaporated under reduced pressure to afford the crude product, which was purified by FC (cyclohexane/EtOAc) affording the pure bisallylated amine.

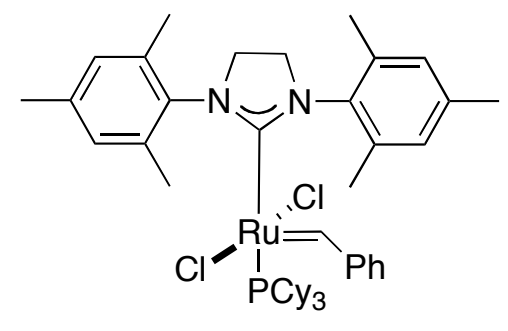

1

Second generation Grubbs’ catalyst

\section{General procedure $C$ : ring closing metathesis of gem-diallylated amines}

Acetyl chloride $(290 \mu \mathrm{L}, 4.07 \mathrm{mmol})$ was added at $0{ }^{\circ} \mathrm{C}$ to $\mathrm{MeOH}(4 \mathrm{~mL})$. The mixture was stirred for $5 \mathrm{~min}$ and the free amine $(3.7 \mathrm{mmol})$ was added. Stirring was continued for $20 \mathrm{~min}$ at room temperature, the solvent was evaporated. The crude oily product was triturated with $\mathrm{Et}_{2} \mathrm{O}$ to afford the amine hydrochloride as pale white solid. The catalyst $1(157 \mathrm{mg}, 0.185 \mathrm{mmol})$ was added to a solution of the amine hydrochloride $(3.7 \mathrm{mmol})$ in $\mathrm{CH}_{2} \mathrm{Cl}_{2}(37 \mathrm{~mL})$. The mixture was heated at 
reflux for $1.5 \mathrm{~h}$ and was treated with $\mathrm{NaOH} 1 \mathrm{M}(10 \mathrm{~mL})$. The aqueous phase was extracted with $\mathrm{CH}_{2} \mathrm{Cl}_{2}$ and the collected organic phases were dried over $\mathrm{MgSO} 4$. Evaporation of the solvent under reduced pressure followed by FC of the residue (cyclohexane/EtOAc) afforded the spirocyclic amine.

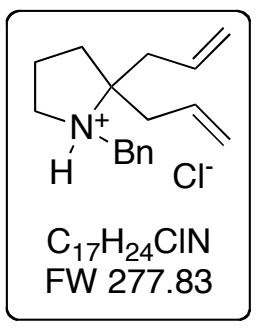

\section{2,2-Diallyl-1-benzylpyrrolidine hydrochloride (4a)}

According general procedure B. Thioiminium ion 3a (1.6 g, $4.8 \mathrm{mmol})$ afforded the free amine $(975 \mathrm{mg}$ ) after FC (cyclohexane/EtOAc 9:1) as yellowish oil. The free amine was treated with $\mathrm{HCl}$ (generated from acetyl chloride and $\mathrm{MeOH}$ ) affording 4a (1.1g, 83\%). Greyish solid. Decomposition $140{ }^{\circ} \mathrm{C} .{ }^{1} \mathrm{H}$ NMR $\left(\mathrm{CDCl}_{3}\right.$, $300 \mathrm{MHz}): 11.98$ (bs, 1H) $7.72(\mathrm{~m}, 2 \mathrm{H}) 7.41(\mathrm{~m}, 3 \mathrm{H}) 5.90(\mathrm{~m}, 2 \mathrm{H}) 5.37$ (m, 4H) 4.53 (B part of $\left.\mathrm{ABX}, \mathrm{J}_{\mathrm{AB}}=12.6, \mathrm{~J}_{\mathrm{BX}}=4 \mathrm{~Hz}, 1 \mathrm{H}\right) 3.92\left(\mathrm{~A}\right.$ part of $\left.\mathrm{ABX}, \mathrm{J}_{\mathrm{AB}}=12.6 \mathrm{~Hz} \mathrm{~J}_{\mathrm{AX}}=8.5 \mathrm{~Hz}, 1 \mathrm{H}\right) 3.55-$ $3.34(\mathrm{~m}, 1 \mathrm{H}) 3.06\left(\mathrm{~B}\right.$ part of $\left.\mathrm{ABX}, \mathrm{J}_{\mathrm{AB}}=14.2, \mathrm{~J}_{\mathrm{BX}}=6 \mathrm{~Hz}, 1 \mathrm{H}\right) 3.01-2.85(\mathrm{~m}, 2 \mathrm{H}) 2.66(\mathrm{~B}$ part of $\left.\mathrm{ABX}, \mathrm{J}_{\mathrm{AB}}=14.2, \mathrm{~J}_{\mathrm{BX}}=6.4 \mathrm{~Hz}, 1 \mathrm{H}\right) 2.55-2.38(\mathrm{~m}, 2 \mathrm{H}) 2.27-2.08(\mathrm{~m}, 2 \mathrm{H}) 1.91(\mathrm{~m}, 1 \mathrm{H}) .{ }^{13} \mathrm{C} \mathrm{NMR}$ $\left(\mathrm{CDCl}_{3}, 75.5 \mathrm{MHz}\right): 131.62,131.15,131.01,129.69,129.52,129.00,121.04,120.80,73.38,52.69$, 51.93, 40.19, 36.47, 33.70, 19.37. IR (diamond ATR) 2480, 1440, 1427, 1007, $925 \mathrm{~cm}^{-1}$. MS $\left(\mathrm{ESI}^{+}\right)$: $242.4\left(100, \mathrm{M}-\mathrm{Cl}^{+}\right), 200.4$ (63). HRMS: calcd for $\mathrm{C}_{17} \mathrm{H}_{24} \mathrm{~N}^{+} 242.1908$ found 242.1898 .

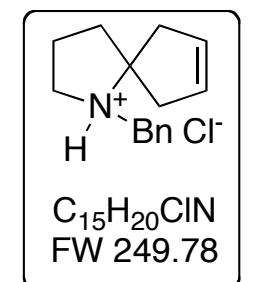

\section{1-Benzyl-1-azaspiro[4.4]non-7-ene hydrochloride (9a)}

According to general procedure $\mathbf{C}$. The amine hydrochloride $4 \mathbf{a}(1.03 \mathrm{~g}, 3.7 \mathrm{mmol})$ afforded the spirocyclic free amine $(777 \mathrm{mg}$ ) after FC (cyclohexane/EtOAc 9:1) as brown oil. The spirocyclic free amine was converted into its hydrochloride by treatment with $\mathrm{HCl}$ (generated from acetyl chloride in $\mathrm{MeOH}$ ) affording 9a (880 mg, 97\% yield). Brown solid. M.p. $=167-168{ }^{\circ} \mathrm{C} .{ }^{1} \mathrm{H} \mathrm{NMR}\left(\mathrm{CDCl}_{3}, 400 \mathrm{MHz}\right): 12.25$ (bs, 1H) 7.74 (m, 2H) 7.37 (m, $3 \mathrm{H}) 5.83(\mathrm{~m}, 1 \mathrm{H}) 5.77(\mathrm{~m}, 1 \mathrm{H}) 4.35\left(\mathrm{~B}\right.$ part of $\left.\mathrm{ABX} \mathrm{J_{ \textrm {AB } }}=13.0 \mathrm{~Hz} \mathrm{~J}_{\mathrm{BX}}=3.6 \mathrm{~Hz}\right), 3.58(\mathrm{~A}$ part of $\left.\mathrm{ABX} \mathrm{J}_{\mathrm{AB}}=13.0 \mathrm{~Hz}, \mathrm{~J}_{\mathrm{AX}}=9.0 \mathrm{~Hz}, 1 \mathrm{H}\right) 3.51(\mathrm{~B}$ part of $\mathrm{AB}, \mathrm{J}=19 \mathrm{~Hz}, 1 \mathrm{H}) 3.20(\mathrm{~m}, 1 \mathrm{H}) 2.88(\mathrm{~m}$, 4H) 2.44 (A part of $\mathrm{AB}, \mathrm{J}=19 \mathrm{~Hz}, 1 \mathrm{H}) 2.09(\mathrm{~m}, 2 \mathrm{H}) 1.94(\mathrm{~m}, 1 \mathrm{H}) .{ }^{13} \mathrm{C} \mathrm{NMR}\left(\mathrm{CDCl}_{3}, 133.3 \mathrm{MHz}\right)$ : 130.97, 130.27, 129.59, 129.43, 128.86, 127.17, 76.81, 51.81, 48.82, 39.76, 39.25, 36.46, 19.22 . IR (diamond ATR) 2444, 1444, $696 \mathrm{~cm}^{-1}$. MS (ESI $\left.{ }^{+}\right): 214.4\left(100, \mathrm{M}^{-\mathrm{Cl}^{+}}\right)$. HRMS: calcd for $\mathrm{C}_{15} \mathrm{H}_{20} \mathrm{~N}^{+}$ 214.1595; found 214.1594. Elemental analysis $\left(\mathrm{C}_{15} \mathrm{H}_{20} \mathrm{NCl}^{+}\right)$: calcd $\mathrm{C} 72.18 \% \mathrm{H} \mathrm{8.01 \%} \mathrm{N} \mathrm{5.61 \% ;}$ found C $71.83 \% \mathrm{H} 8.16 \% \mathrm{~N} 5.54 \%$. 


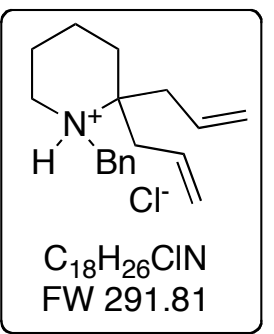

2,2-diallyl-1-benzylpiperidine hydrochloride (4b)

According to general procedure $\mathbf{B}$. Thiominium ion $\mathbf{3 b}(1.74 \mathrm{~g}, 5 \mathrm{mmol})$ afforded free amine (1.24 g) after FC (cyclohexane/EtOAc 95:5) as colourless oil. The free amine was then treated with $\mathrm{HCl}$ (generated from acetyl chloride and $\mathrm{MeOH}$ ) affording 4b (1.41 g, 97\%). White solid. M.p. $=152-154^{\circ} \mathrm{C} .{ }^{1} \mathrm{H}$ NMR $\left(\mathrm{CDCl}_{3}\right.$, $400.13 \mathrm{MHz}): 12.79$ (bs, 1H) $7.72(\mathrm{~m}, 2 \mathrm{H}) 7.40(\mathrm{~m}, 3 \mathrm{H}) 5.97(\mathrm{~m}, 2 \mathrm{H})$ 5.35-5.25 (m, 4H) 4.78 (B part of $\left.\mathrm{ABX}, \mathrm{J}_{\mathrm{AB}}=13.0 \mathrm{~Hz}, \mathrm{~J}_{\mathrm{BX}}=4 \mathrm{~Hz}, 1 \mathrm{H}\right) 3.84\left(\mathrm{~A}\right.$ part of $\mathrm{ABX}, \mathrm{J}_{\mathrm{AB}}=13.0 \mathrm{~Hz}, \mathrm{~J}_{\mathrm{AX}}=9 \mathrm{~Hz}, 1 \mathrm{H}$ ) $3.23\left(\mathrm{~B}\right.$ part of $\left.\mathrm{ABX}, \mathrm{J}_{\mathrm{AB}}=14.0 \mathrm{~Hz}, \mathrm{~J}_{\mathrm{BX}}=7.2 \mathrm{~Hz}, 1 \mathrm{H}\right) 3.16-3.03(\mathrm{~m}, 2 \mathrm{H}) 2.85-2.71(\mathrm{~m}, 3 \mathrm{H}) 2.46-$ $2.38(\mathrm{~m}, 1 \mathrm{H}) \quad 2.32-2.10(\mathrm{~m}, 1 \mathrm{H}) \quad 1.85-1.60(\mathrm{~m}, 1 \mathrm{H})$ 1.74-1.54 (m, 3H). ${ }^{13} \mathrm{C} \mathrm{NMR}\left(\mathrm{CDCl}_{3}, 133\right.$ MHz): 131.82, 131.51, 130.71, 129.67, 128.97, 128.82, 66.84, 53.20, 46.33, 40.67, 34.42, 31.20, 21.38, 17.83. IR (diamond ATR) 2525, 1462, $1428 \mathrm{~cm}^{-1}$. MS (ESI $\left.{ }^{+}\right): 256.4\left(100,{\mathrm{M}-\mathrm{Cl}^{+}}^{+}\right), 242.4$ (19). HRMS: calcd for $\mathrm{C}_{18} \mathrm{H}_{26} \mathrm{~N}^{+} 256.2056$; found 256.2056.

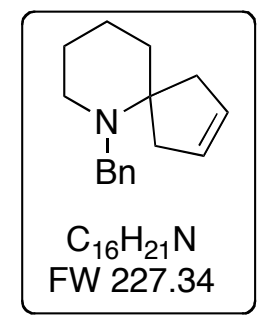

\section{6-Benzyl-6-azaspiro[4.5]dec-2-ene (9b)}

According to general procedure $\mathbf{C}$ The amine hydrochloride $4 \mathbf{b}(50 \mathrm{mg}, 0.17$ mmol) afforded amine $\mathbf{8 b} \quad(32 \mathrm{mg}, 82 \%$ yield $)$. Brownish oil. FC (cyclohexane/EtOAc 95:5). ${ }^{1} \mathrm{H}$ NMR $\left(\mathrm{CDCl}_{3}, 300 \mathrm{MHz}\right):$ 7.27-7.07 (m, 5H) 5.58 $(\mathrm{s}, 2 \mathrm{H}) 3.23(\mathrm{~s}, 2 \mathrm{H}) 2.52(\mathrm{~m}, 2 \mathrm{H}) 2.27(\mathrm{~m}, 2 \mathrm{H}) 2.11(\mathrm{~m}, 2 \mathrm{H}) 1.61(\mathrm{~m}, 2 \mathrm{H}) 1.40(\mathrm{~m}$,

$2 \mathrm{H}) \cdot{ }^{13} \mathrm{C} \mathrm{NMR}\left(\mathrm{CDCl}_{3}, 75.5 \mathrm{MHz}\right): 141.32,129.44,128.47,128.16,126.46,65.25,54.70,48.37$, 40.66, 26.42, 22.17 . IR (diamond ATR): 2924, 2848, 2789, 1451, $1430 \mathrm{~cm}^{-1}$. MS (ESI $\left.{ }^{+}\right): 228\left(\mathrm{M}^{+}\right.$, 100\%), 215 (40\%). HRMS: calcd for $\mathrm{C}_{16} \mathrm{H}_{22} \mathrm{~N}^{+} 228.1752$; found 228.1746 .

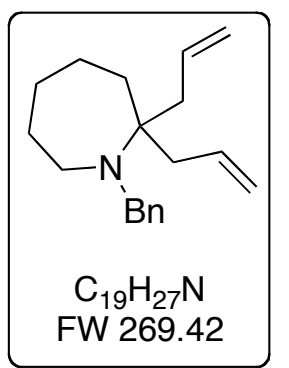

\section{2,2-Diallyl-1-benzylazepane (4c)}

According to general procedure B. Thioiminiun ion 3c $(180 \mathrm{mg}, 0.5 \mathrm{mmol})$ afforded 4c (113 mg, 85\%). Viscous oil. FC (cyclohexane/EtOAc 9:1). ${ }^{1} \mathrm{H}$ NMR $\left(\mathrm{CDCl}_{3}, 400 \mathrm{MHz}\right): 7.39(\mathrm{~m}, 2 \mathrm{H}) 7.30(\mathrm{~m}, 2 \mathrm{H})$ 7.23-7.18 (m, 1H) 6.02-5.92 (m, 2H) 5.08-5.03 (m, 4H) $3.81(\mathrm{~s}, 2 \mathrm{H}) 2.64(\mathrm{~m}, 2 \mathrm{H}) 2.43\left(\mathrm{~B}\right.$ part of $\mathrm{ABX}, \mathrm{J}_{\mathrm{AB}}=14.0$ $\left.\mathrm{Hz}, \mathrm{J}_{\mathrm{BX}}=7.2 \mathrm{~Hz}, 2 \mathrm{H}\right) 2.30\left(\mathrm{~A}\right.$ part of $\left.\mathrm{ABX}, \mathrm{J}_{\mathrm{AB}}=14.0 \mathrm{~Hz}, \mathrm{~J}_{\mathrm{AX}}=7.2 \mathrm{~Hz}, 2 \mathrm{H}\right)$ $1.68(\mathrm{~m}, 4 \mathrm{H}) 1.43(\mathrm{~m}, 2 \mathrm{H}) 1.30(\mathrm{~m}, 2 \mathrm{H}) .{ }^{13} \mathrm{C} \mathrm{NMR}\left(\mathrm{CDCl}_{3}, 100 \mathrm{MHz}\right): 142.28,136.09,128.45$, 127.94, 126.40, 116.88, 61.19, 54.81, 48.73, 42.84, 37.19, 31.87, 29.98, 22.74. IR (diamond ATR): 3072, 2925, 1636, $1440 \mathrm{~cm}^{-1}$. MS (ESI $\left.{ }^{+}\right): 270.4\left(100, \mathrm{M}+\mathrm{H}^{+}\right), 230.4$ (14), 180.2 (83). HRMS: calcd for $\mathrm{C}_{19} \mathrm{H}_{28} \mathrm{~N}^{+}$270.2221; found: 270.2221. 


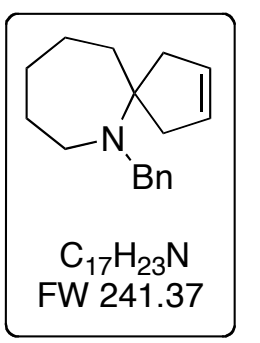

\section{6-Benzyl-6-azaspiro[4.6]undec-2-ene (9c)}

According general procedure C. Bisallylated amine 4c (113 mg, $0.47 \mathrm{mmol})$ afforded 8c (28 mg, 83\%). Greenish oil. FC (cyclohexane/EtOAc 95:5). ${ }^{1} \mathrm{H}$ NMR $\left(\mathrm{CDCl}_{3}, 300 \mathrm{MHz}\right): 7.42$ (d, J = 7.2 Hz, 2H) $7.29(\mathrm{~m}, 3 \mathrm{H}) 7.20(\mathrm{~m}, 1 \mathrm{H}) 5.58(\mathrm{~s}, 2 \mathrm{H})$ $3.42(\mathrm{~s}, 2 \mathrm{H}) 2.58(\mathrm{~m}, 4 \mathrm{H}) 2.23(\mathrm{dm}, \mathrm{J}=14.5 \mathrm{~Hz}, 2 \mathrm{H}) 1.80(\mathrm{~m}, 2 \mathrm{H}) 1.69(\mathrm{~m}, 2 \mathrm{H})$ $1.52(\mathrm{~m}, 2 \mathrm{H}) 1.41(\mathrm{~m}, 2 \mathrm{H}) .{ }^{13} \mathrm{C} \mathrm{NMR}\left(\mathrm{CDCl}_{3}, 75.5 \mathrm{MHz}\right): 142.3,129.6,128.6,128.1,126.5,68.4$, 55.6, 48.5, 44.1, 43.9, 31.4, 29.8, 23.1. IR (diamond ATR): 3046, 2923, 2848, 1450, $1439 \mathrm{~cm}^{-1}$. MS (EI $\left.{ }^{+}\right): 241\left(26, \mathrm{M}^{+}\right), 212$ (48), 136 (36), 96 (47), 91 (100). HRMS: calcd for $\mathrm{C}_{17} \mathrm{H}_{24} \mathrm{~N}^{+} 242.1908$; found: 242.1905 .

\section{General procedure D: synthesis of gem-dibenzylated amines}

Benzyl magnesium chloride $(2 \mathrm{~mL}, 2.0 \mathrm{mmol})$ was added to a suspension of thioiminium ion (1.0 $\mathrm{mmol})$ in dry THF $(10 \mathrm{ml})$ at room temperature. After $10 \mathrm{~min}$ a solid was formed and the mixture was stirred for $10 \mathrm{~h}$ at room temperature, treated with saturated solution of ammonium chloride. The aqueous phase was extracted with EtOAc, the collected organic phase were washed with brine and dried over $\mathrm{MgSO}_{4}$. Evaporation of the solvent at reduced pressure afforded a crude product which was purified by FC (cyclohexane/EtOAc).

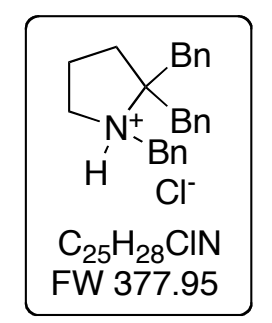

\section{2,2-Dibenzyl-N-benzylpyrrolidine hydrochloride (5a)}

According to general procedure starting D. Thioiminium ion 3a $(333 \mathrm{mg}, 1 \mathrm{mmol})$ afforded free amine (280 mg) after FC (cyclohexane/EtOAc 9:1) as brown oil. The free amine was converted into its hydrochloride by treatment with $\mathrm{HCl}$ (generated from $\mathrm{AcCl}$ in $\mathrm{MeOH}$ ) affording 5a (314 mg, 92\%). Pink solid. Decomposition 170

${ }^{\circ} \mathrm{C} .{ }^{1} \mathrm{H}$ NMR $\left(\mathrm{CDCl}_{3}, 400 \mathrm{MHz}\right): 12.35(\mathrm{~s}, 1 \mathrm{H}) 7.71\left(\mathrm{dd}, \mathrm{J}_{1}=8.0 \mathrm{~Hz}, \mathrm{~J}_{2}=2.0 \mathrm{~Hz}, 2 \mathrm{H}\right) 7.61(\mathrm{~d}, \mathrm{~J}=$ $8.0 \mathrm{~Hz}, 2 \mathrm{H}) 7.35(\mathrm{~m}, 11 \mathrm{H}) 4.29(\mathrm{~B}$ part of $\mathrm{AB}, \mathrm{J}=12.4,3.4 \mathrm{~Hz}, 1 \mathrm{H}) 3.97$ (A part of $\mathrm{AB}, \mathrm{J}=12.6$, $8.7 \mathrm{~Hz}, 1 \mathrm{H}) 3.84\left(\mathrm{~B}\right.$ part of $\left.\mathrm{ABX}, \mathrm{J}_{\mathrm{AB}}=13.7 \mathrm{~Hz}, 1 \mathrm{H}\right) 3.43\left(\mathrm{~A}\right.$ part of $\left.\mathrm{ABX}, \mathrm{J}_{\mathrm{AB}}=13.7 \mathrm{~Hz}, 1 \mathrm{H}\right) 3,38$ $(\mathrm{m}, 1 \mathrm{H}) 3.28$ (B part of $\mathrm{AB}, \mathrm{J}=14.6 \mathrm{~Hz}, 1 \mathrm{H}) 3.07$ (A part of $\mathrm{AB}, \mathrm{J}=14.5 \mathrm{~Hz}, 1 \mathrm{H}) 2.80-2.62(\mathrm{~m}, 1$ H) $2.68-2.51(\mathrm{~m}, 1 \mathrm{H}) .{ }^{13} \mathrm{C} \mathrm{NMR}\left(\mathrm{CDCl}_{3}, 133.33 \mathrm{MHz}\right)$ : 135.80, 135.35, 131.29, 130.48, 130.01, $129.90,129.35,129.30,129.05,128.20,127.92,75.69,52.78,52.34,42.44,37.56,34.46,19.73$. IR (diamond ATR): 3469, 3387, 1633, 1602, 1495, $1462 \mathrm{~cm}^{-1}$. Elemental analysis $\left(\mathrm{C}_{25} \mathrm{H}_{28} \mathrm{NCl} 0.6\right.$ $\mathrm{H}_{2} \mathrm{O}$ ): calcd C 77.28\% H 7.52\% N 3.60\%; found C 77.27\% H 7.53\% N 3.65\%. 


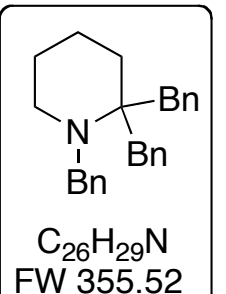

FW 355.52

\section{2,2-Dibenzyl-N-benzylpiperidine (5b)}

According to general procedure D. Thioiminium ion $\mathbf{3 b}(100 \mathrm{mg}, 0.29 \mathrm{mmol})$ afforded 5b (89 mg, 86\%) Orange glue. FC (cyclohexane/EtOAc 9:1). ${ }^{1} \mathrm{H}$ NMR $\left(\mathrm{CDCl}_{3}, 300 \mathrm{MHz}\right): 7.28-7.15(\mathrm{~m}, 5 \mathrm{H}) 4.02(\mathrm{~s}, 2 \mathrm{H}) 3.30(\mathrm{~d}, \mathrm{~J}=13.6 \mathrm{~Hz}, 2 \mathrm{H}) 2.58$ $(\mathrm{d}, \mathrm{J}=13.6 \mathrm{~Hz}, 2 \mathrm{H}) 2.52(\mathrm{t}, \mathrm{J}=5.8 \mathrm{~Hz}, 2 \mathrm{H}) 1.67(\mathrm{~m}, 2 \mathrm{H}) 1.48(\mathrm{~m}, 2 \mathrm{H}) 1.38(\mathrm{~m}$, 2H). ${ }^{13} \mathrm{C} \mathrm{NMR}\left(\mathrm{CDCl}_{3}, 75.5 \mathrm{MHz}\right)$ : 139.39, 137.90, 130.32, 127.33, 127.16, 126.78, 125.47, 124.93, 59.92, 52.50, 45.84, 37.78, 29.87, 25.14, 20.41. IR (diamond ATR): 2914, 1492, $1454 \mathrm{~cm}^{-1} 1$. MS (ESI $\left.{ }^{+}\right)$: $356.23\left(100, \mathrm{M}+\mathrm{H}^{+}\right), 273.17$ (33), 215.13 (83). HRMS: calcd for $\mathrm{C}_{26} \mathrm{H}_{30} \mathrm{~N}^{+} 356.2374$; found: 356.2376 .

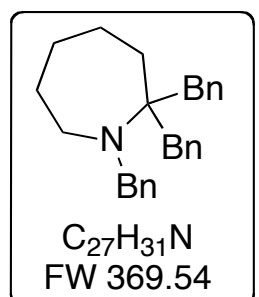

\section{2,2-dibenzyl-N-benzylazepine (5c)}

According to general procedure D. Thioiminium ion 3c $(100 \mathrm{mg}, 0.28 \mathrm{mmol})$ afforded 5c (78 mg, 79 \%). White oil. FC (cyclohexane/EtOAc 9:1). ${ }^{1} \mathrm{H}$ NMR $\left(\mathrm{CDCl}_{3}, 300 \mathrm{MHz}\right): 7.57(\mathrm{~d}, \mathrm{~J}=7.2 \mathrm{~Hz}, 2 \mathrm{H})$ 7.42-7-25 (m, 13H) $4.31(\mathrm{~s}, 2 \mathrm{H}) 3.17$ $(\mathrm{d}, \mathrm{J}=13.6 \mathrm{~Hz}, 2 \mathrm{H}) 2.80(\mathrm{~d}, \mathrm{~J}=13.6 \mathrm{~Hz}, 2 \mathrm{H}) 2.41(\mathrm{~m}, 2 \mathrm{H}) 1.69(\mathrm{~m}, 4 \mathrm{H}) 1.32(\mathrm{~m}$,

2H) $1.22(\mathrm{~m}, 2 \mathrm{H}) .{ }^{13} \mathrm{C} \mathrm{NMR}\left(\mathrm{CDCl}_{3}, 75.5 \mathrm{MHz}\right): 142.74,139.39,131.43,128.47,128.16,127.88$, 126.60, 126.01, 63.61, 56.05, 50.42, 43.89, 35.00, 32.49, 30.24, 23.27. IR (diamond ATR): 2947, 2926, 1493, $1453 \mathrm{~cm}^{-1} . \mathrm{MS}\left(\mathrm{ESI}^{+}\right)$: 350.25 (100, M+H $\left.{ }^{+}\right), 353.27$ (31), 273.17 (18), 215.13 (59). HRMS: calcd for $\mathrm{C}_{27} \mathrm{H}_{32} \mathrm{~N} 370.2539$; found: 370.2534 .

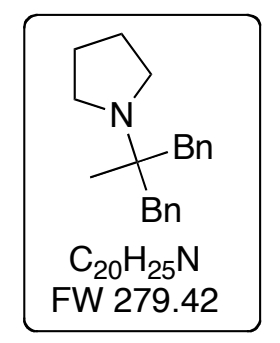

\section{1-(1-Benzyl-1-methyl-2-phenyl-ethyl)-pyrrolidine (8)}

According general procedure D. Thioiminium ion $7(0.277 \mathrm{~g}, 1.02 \mathrm{mmol})$ afforded $8(0.253 \mathrm{~g}, 87 \%)$. Transparent oil. FC (cyclohexane/EtOAc 9:1). ${ }^{1} \mathrm{H} \mathrm{NMR}\left(\mathrm{CDCl}_{3}\right.$, $300 \mathrm{MHz})$ : 7.27-7.20 (m, 10H) $2.94(\mathrm{~B}$ part of $\mathrm{AB}, \mathrm{J}=13.5 \mathrm{~Hz}, 2 \mathrm{H}) 2.85(\mathrm{~m}, 4 \mathrm{H})$ 2.70 (A part of $\mathrm{AB}, \mathrm{J}=13.5 \mathrm{~Hz}, 2 \mathrm{H}) 1.78(\mathrm{~m}, 4 \mathrm{H}) 0.91(\mathrm{~s}, 3 \mathrm{H}) .{ }^{13} \mathrm{C} \mathrm{NMR}\left(\mathrm{CDCl}_{3}\right.$, 75.5 MHz): 139.52, 130.93, 127.79, 125.81, 58.67, 45.37, 41.71, 24.36, 23.06. IR (diamond ATR):

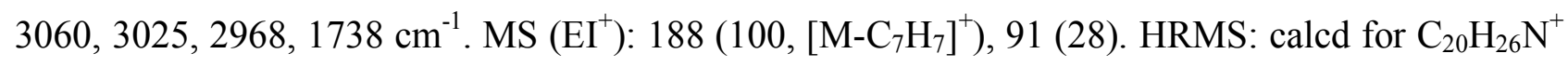
280.2065; found: 280.2072 .

\section{General procedure E: synthesis of gem-dialkylated amines}

A suspension of $\mathrm{CeCl}_{3}(0.296 \mathrm{~g} .1 .2 \mathrm{mmol})$ in dry THF $(4 \mathrm{ml})$ was sonicated at room temperature for $30 \mathrm{~min}$. The suspension was cooled down to $-78^{\circ} \mathrm{C}$ and the alkyllithium reagent solution $(1.2$ mmol) was added dropwise. The solution became pale yellow and stirring was continued for 30 
min. The thioiminium salt was added as a solid $(0.3 \mathrm{mmol})$, the cooling bath was removed and the mixture was stirred for $6 \mathrm{~h}$. The dark brown suspension was treated with saturated $\mathrm{NH}_{4} \mathrm{Cl}$ and the aqueous phases were extracted with dichloromethane $(3 \mathrm{x})$. The collected organic phases were dried over $\mathrm{MgSO}_{4}$, filtrated and the solvent evaporated under reduced pressure. The crude product was purified by FC (cyclohexane/tert-BuOMe) to afford the gem-dialkylated amine.

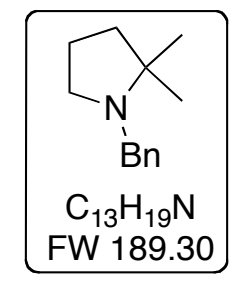

\section{1-Benzyl-2,2-dimethylpyrrolidine (10a) ${ }^{1}$}

According general procedure E. Thioiminium ion 3a $(0.200 \mathrm{~g}, 0.74 \mathrm{mmol})$ afforded 9a $(0.088 \mathrm{~g}, 77 \%)$. Transparent oil. FC (cyclohexane/tert-BuOMe 85:15). ${ }^{1} \mathrm{H}$ NMR 4H) $1.01(\mathrm{~s}, 6 \mathrm{H}) .{ }^{13} \mathrm{C} \mathrm{NMR}\left(\mathrm{CDCl}_{3}, 75.5 \mathrm{MHz}\right)$ : 141.09, 128.57, 128.133, 126.50, 76.60, 67.99, 60.05, 53.21, 50.94, 39.98, 29.72, 25.64, 23.07, 20.50. IR (diamond ATR): 3025, 2958, 2866, 1453 $\mathrm{cm}^{-1}$. MS (EI ${ }^{+}$): 189(9), 185(14), 174 (59). HRMS: calcd for $\mathrm{C}_{13} \mathrm{H}_{20} \mathrm{~N}^{+}$190.1599; found 190.1595 .

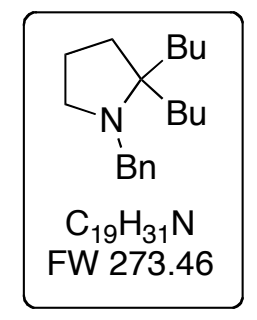

\section{1-Benzyl-2,2-dibutylpyrrolidine (11a)}

According general procedure E. Thioiminium ion 3a $(0.200 \mathrm{~g}, 0.74 \mathrm{mmol})$ afforded $11 \mathrm{a}(0.144 \mathrm{~g}, 88 \%)$ ). Pale yellow oil. FC (cyclohexane/tert-BuOMe 9:1). ${ }^{1} \mathrm{H}$ NMR $\left(\mathrm{CDCl}_{3}, 300 \mathrm{MHz}\right): 7.27-7.24(\mathrm{~m}, 5 \mathrm{H}) 3.52(\mathrm{~s}, 2 \mathrm{H}) 2.53-2.51(\mathrm{t}, \mathrm{J}=6 \mathrm{~Hz}, 2 \mathrm{H}) 1.59$ $1.56(\mathrm{~m}, 4 \mathrm{H}) 1.35-1.12(\mathrm{~m}, 14 \mathrm{H}) 0.85(\mathrm{t}, \mathrm{J}=6 \mathrm{~Hz}, 6 \mathrm{H}) \cdot{ }^{13} \mathrm{C} \mathrm{NMR}\left(\mathrm{CDCl}_{3}, 75.5\right.$ MHz): 141.50, 128.30, 128.09, 126.35, 76.59, 67.98, 64.52, 52.22, 50.76, 35.18, 32.98, 26.81, 25.63, 23.78, 21.82, 14.27. IR (diamond ATR): 3026, 2954, 2858, $1454 \mathrm{~cm}^{-1} . \mathrm{MS}\left(\mathrm{EI}^{+}\right): 274(65)$, 215(28). HRMS: calcd for $\mathrm{C}_{19} \mathrm{H}_{32} \mathrm{~N}^{+}$274.2534; found 274.2541 .

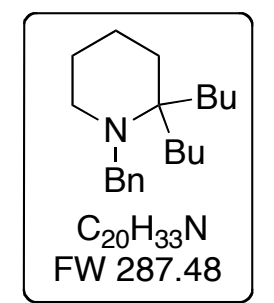

\section{1-Benzyl-2,2-dibutylpiperidine (11b)}

According general procedure E. Thioiminium ion $\mathbf{3 b}(0.078 \mathrm{~g}, 0.29 \mathrm{mmol})$ afforded 11b $(0.057 \mathrm{~g}, 69 \%)$. Colourless oil. FC (cyclohexane/tert-BuOMe 9:1). ${ }^{1} \mathrm{H}$ NMR $\left(\mathrm{CDCl}_{3}, 300 \mathrm{MHz}\right): 7.31-7.14(\mathrm{~m}, 5 \mathrm{H}) 3.45(\mathrm{~s}, 2 \mathrm{H})$ 2.33-2.29 (m, 2H) 1.66-1.65 (m, 2H) $1.43-1.22(\mathrm{~m}, 20 \mathrm{H})$ 0.83-0.81 (m, 8H). ${ }^{13} \mathrm{C} \mathrm{NMR}\left(\mathrm{CDCl}_{3}, 75.5 \mathrm{MHz}\right): 128.48$, $128.08,127.90,126.21,126.18,67.98,60.11,57.50,54.29,52.77,48.28,45.82,38.13,37.09$, $33.30,31.58,30.06,27.07,26.36,25.70,25.63,23.95,23.80,23.11,20.81,14.26,14.23$. IR (diamond ATR): 2930, 2858, 2793, $1452 \mathrm{~cm}^{-1}$. MS $\left(\mathrm{EI}^{+}\right): 288,273,215$. HRMS: calcd for $\mathrm{C}_{20} \mathrm{H}_{34} \mathrm{~N}^{+}$ 288.2691; found 288.2687.

\footnotetext{
${ }^{1}$ Denton, M. S.; Wood, A. Synlett 1999, 55-56
} 


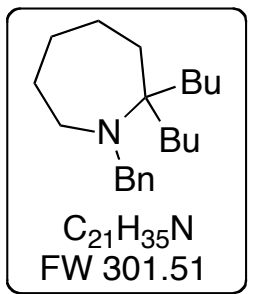

\section{1-Benzyl-2,2-dibutylazepane (11c)}

According general procedure E. Thioiminium ion 3c $(0.100 \mathrm{~g}, 0.28 \mathrm{mmol})$ afforded 11c (0.062 g, $71 \%)$. Colourless oil. FC (cyclohexane/tert-BuOMe 95:5). ${ }^{1} \mathrm{H}$ NMR $\left(\mathrm{CDCl}_{3}, 300 \mathrm{MHz}\right):$ 7.36-7.12 (m, 5H) $3.65(\mathrm{~s}, 2 \mathrm{H}) 2.56(\mathrm{t}, \mathrm{J}=3 \mathrm{~Hz}, 2 \mathrm{H})$ 1.58-1.22 (m, 21H) $0.84(\mathrm{t}, \mathrm{J}=9 \mathrm{~Hz}, 6 \mathrm{H}) \cdot{ }^{13} \mathrm{C} \mathrm{NMR}\left(\mathrm{CDCl}_{3}, 100 \mathrm{~Hz}\right): 142.94$, $128.48,127.90,126.21,60.12,54.29,48.28,38.13,37.09,31.60,30.06,29.72$, 27.07, 23.96, 23.11, 14.27 . IR (diamond ATR): 2923, 2853, 1493, $1455 \mathrm{~cm}^{-1}$. MS (EI $\left.{ }^{+}\right): 302,273,215,212$. HRMS: calcd for $\mathrm{C}_{21} \mathrm{H}_{36} \mathrm{~N}^{+} 302.2847$; found 302.2848.

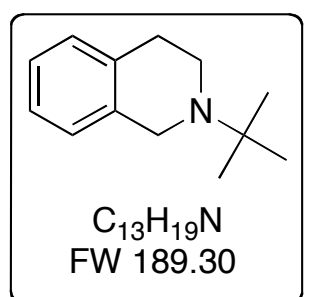

\section{2-tert-Butyl-1,2,3,4-tetrahydroisoquinoline (14)}

According general procedure E. Thioiminium ion $13(0.100 \mathrm{~g}, 0.3 \mathrm{mmol})$ afforded 14 (0.047g, 83\%). Yellow liquid. . FC (cyclohexane/tert-BuOMe 8:2). ${ }^{1} \mathrm{H}$ NMR $\left(\mathrm{CDCl}_{3}, 300 \mathrm{MHz}\right): 7.02(\mathrm{~m}, 4 \mathrm{H}) 3.72(\mathrm{~s}, 2 \mathrm{H})$ 2.79-2.73 (m, 4H) 1.11 $(\mathrm{s}, 9 \mathrm{H}) \cdot{ }^{13} \mathrm{C} \mathrm{NMR}\left(\mathrm{CDCl}_{3}, 75.5 \mathrm{MHz}\right): 134.93,128.77,127.07,125.92,125.57$, 54.06, 49.19, 44.26, 30.71, 26.05. IR (diamond ATR): 2968, 2916, 1585, $1454 \mathrm{~cm}^{-1}$. MS (EI $\left.{ }^{+}\right): 190$, 188, 134, 132. HRMS: calcd for $\mathrm{C}_{13} \mathrm{H}_{20} \mathrm{~N}^{+}$190.1596; found 190.1593 . 
IZ"
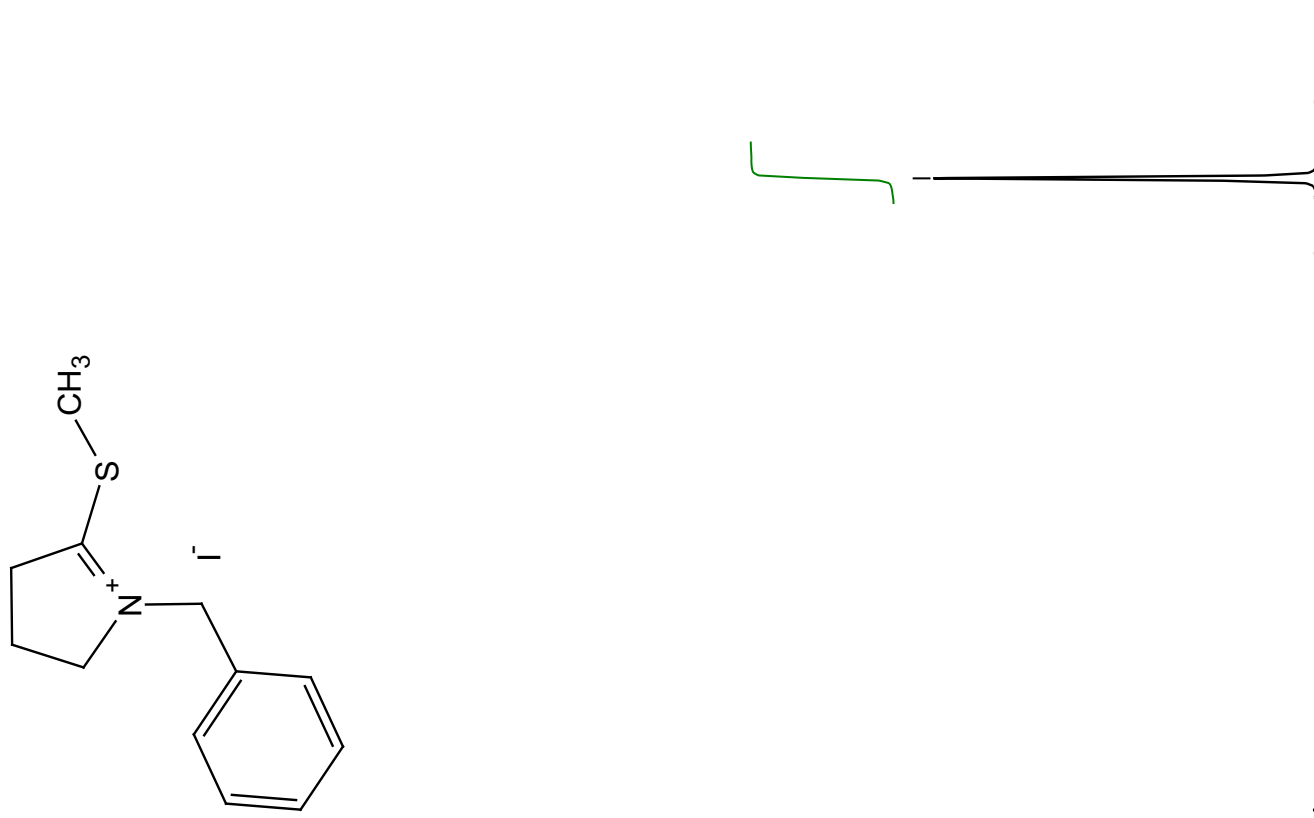
$9 S^{\circ} \angle I-$

S0.6I-

90'It—

80's -

ع0"6ZI

$0 \varepsilon^{\prime} 6 Z \mathrm{I}$

0t'6ZI-

$66^{\circ} 62 \mathrm{II}$

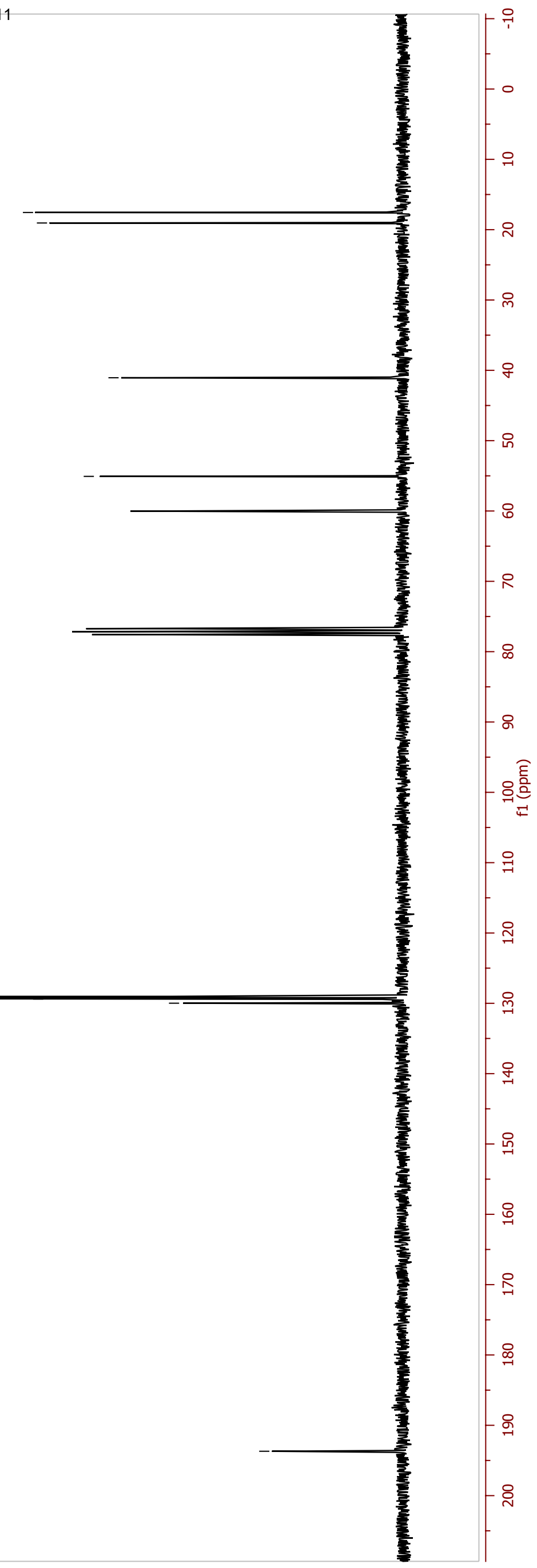




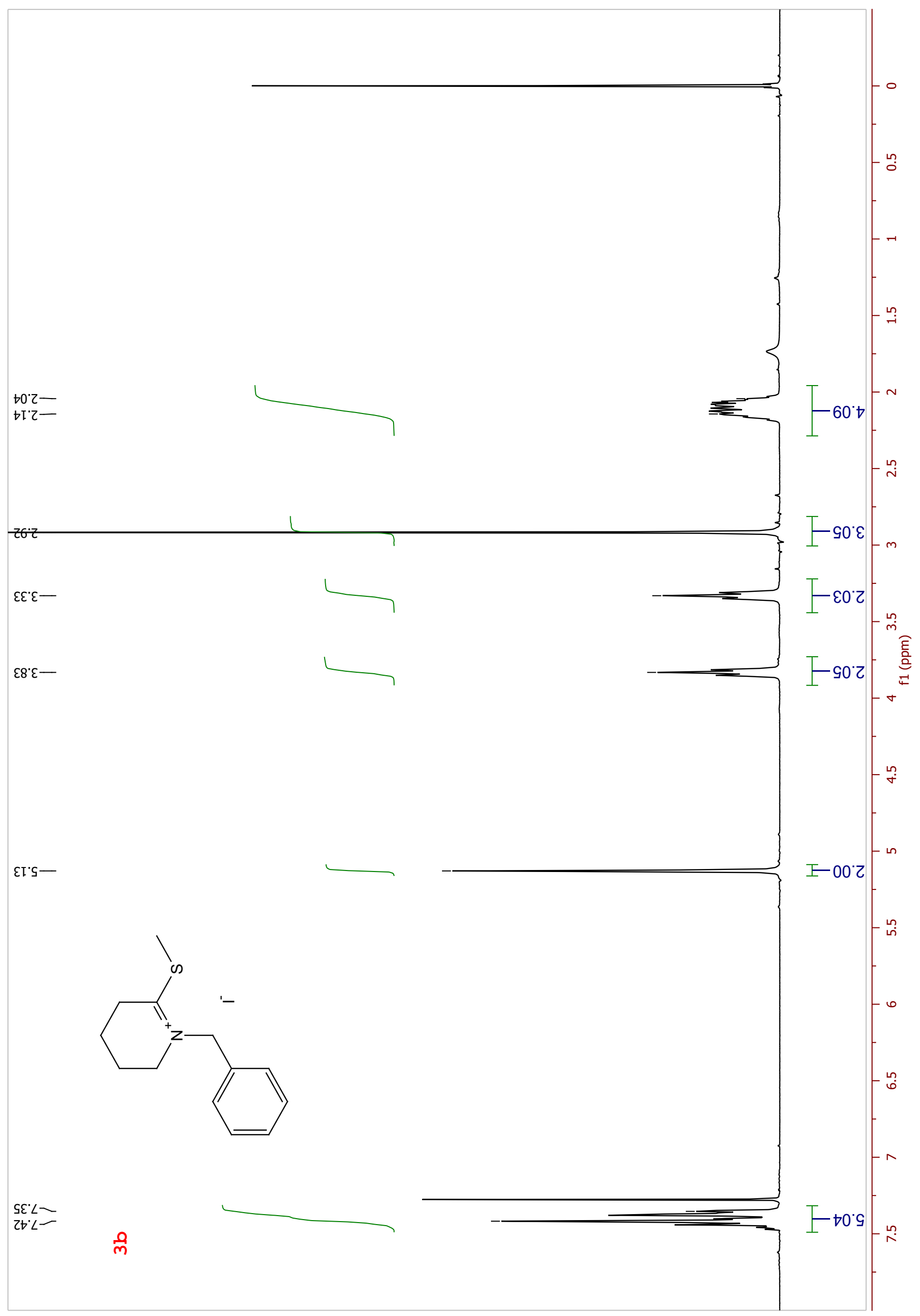


66.91 工

${ }^{\circ} \cdot 8 \mathrm{I}-$

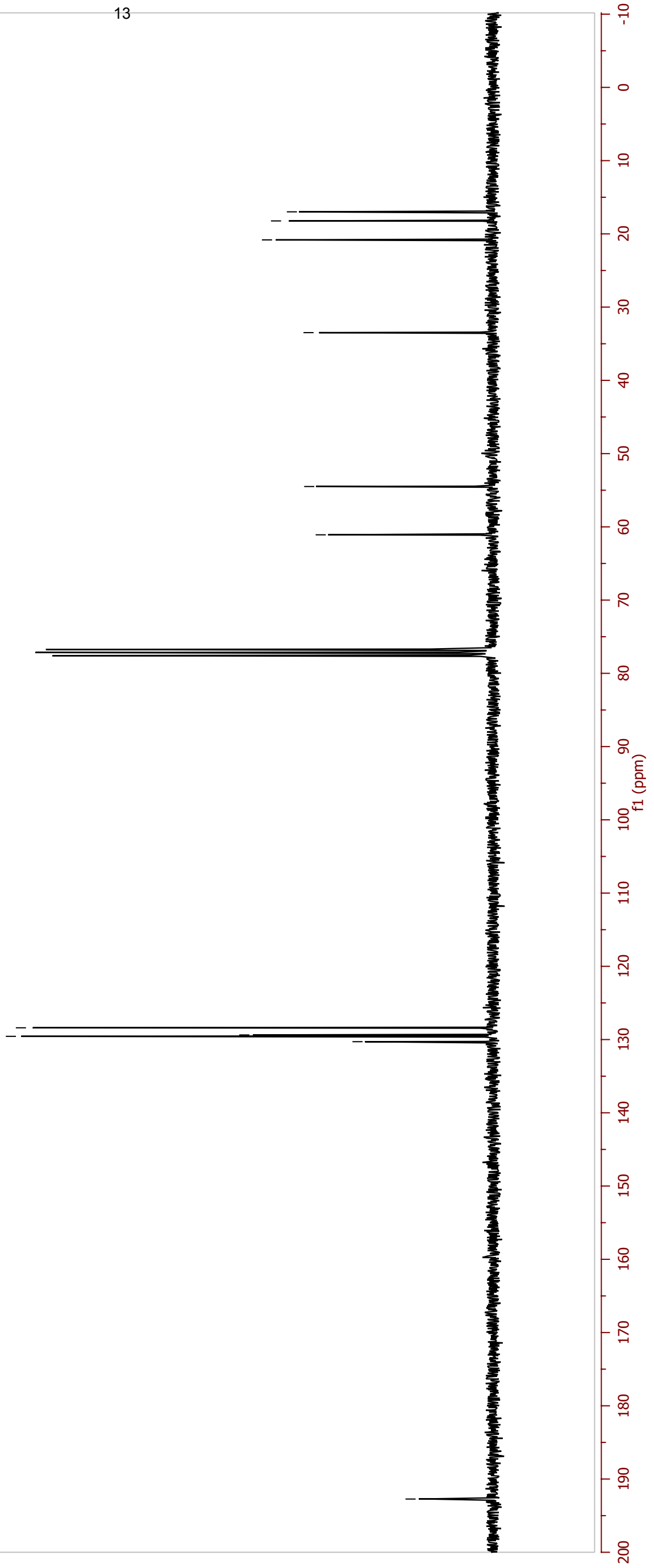

$06.82 \mathrm{I}$

$\left.\angle \varepsilon^{\prime} 6 Z I\right]$

6Z'0हI-

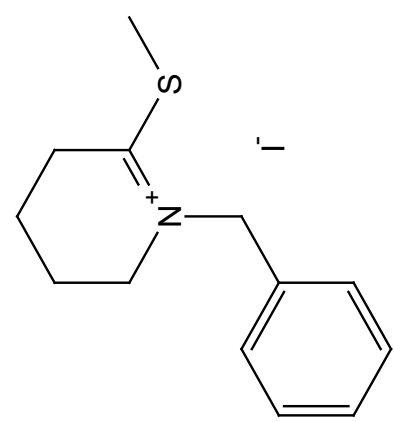

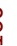

ले 
$56 \cdot 2$

t $\varepsilon-$

9I'b

$8 \mathrm{I}^{\prime} \mathrm{b}$

$\angle I ' S-$

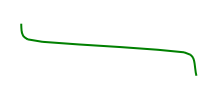

$\downarrow \varepsilon^{\prime} L \backslash$<smiles>COC1=[Z](Cc2ccccc2)CCCCC1</smiles>
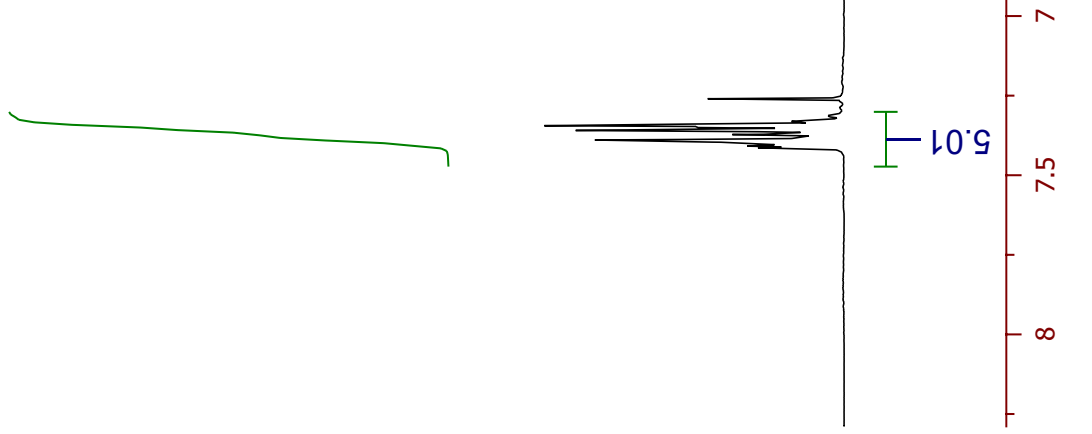
26.81
72.22

s6.t2

OI' 82 -

$\downarrow 6^{\circ} \downarrow \varepsilon-$

95' $85-$

60'29-

โ8.82

OS.6ZI L

$\angle \varepsilon^{\prime} 0 \varepsilon{ }^{\top}$

$S 6^{\circ} \angle 6 I-$

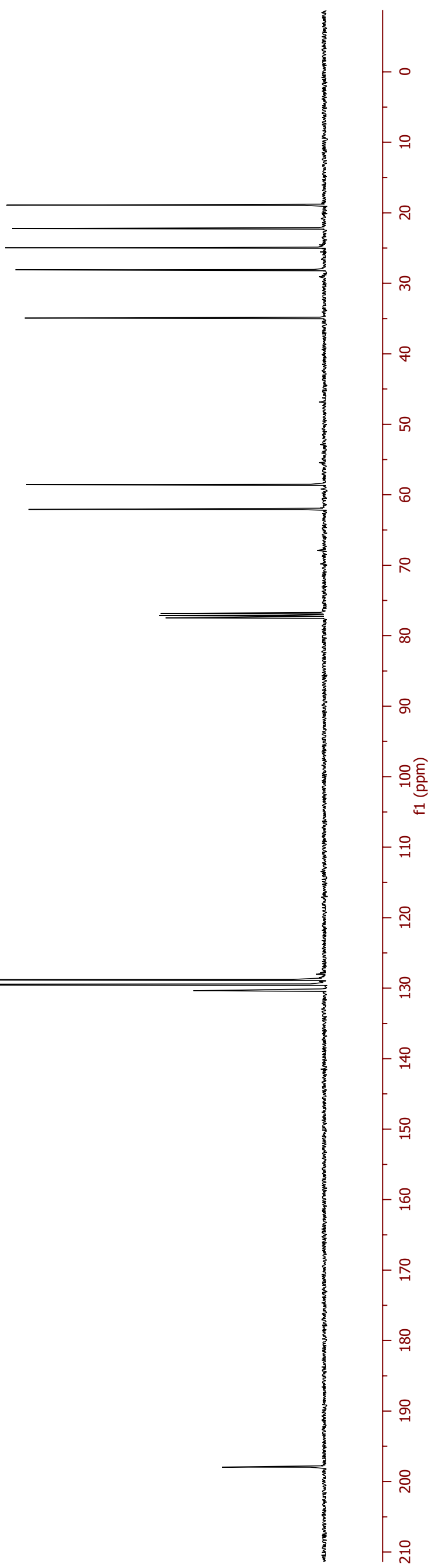




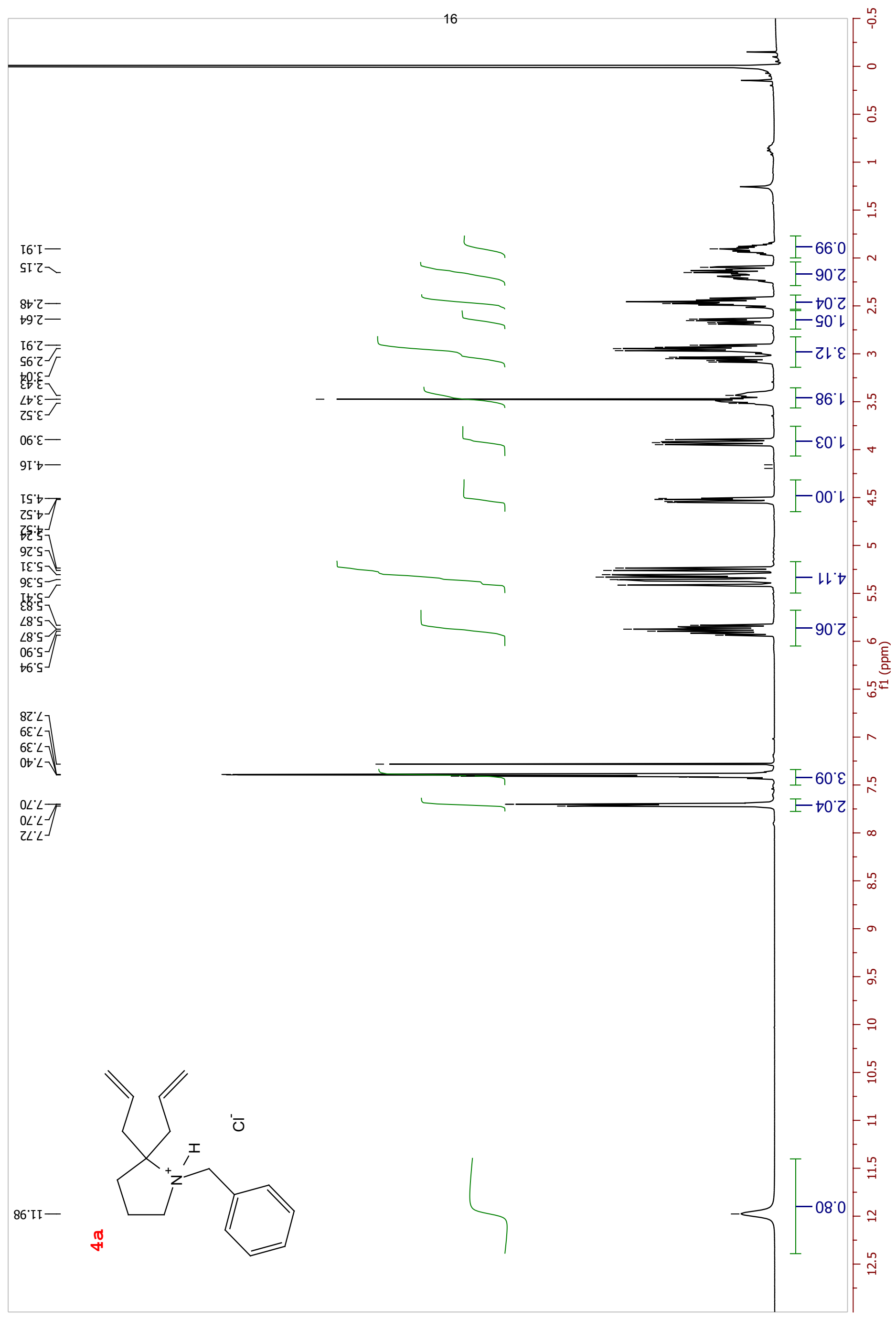



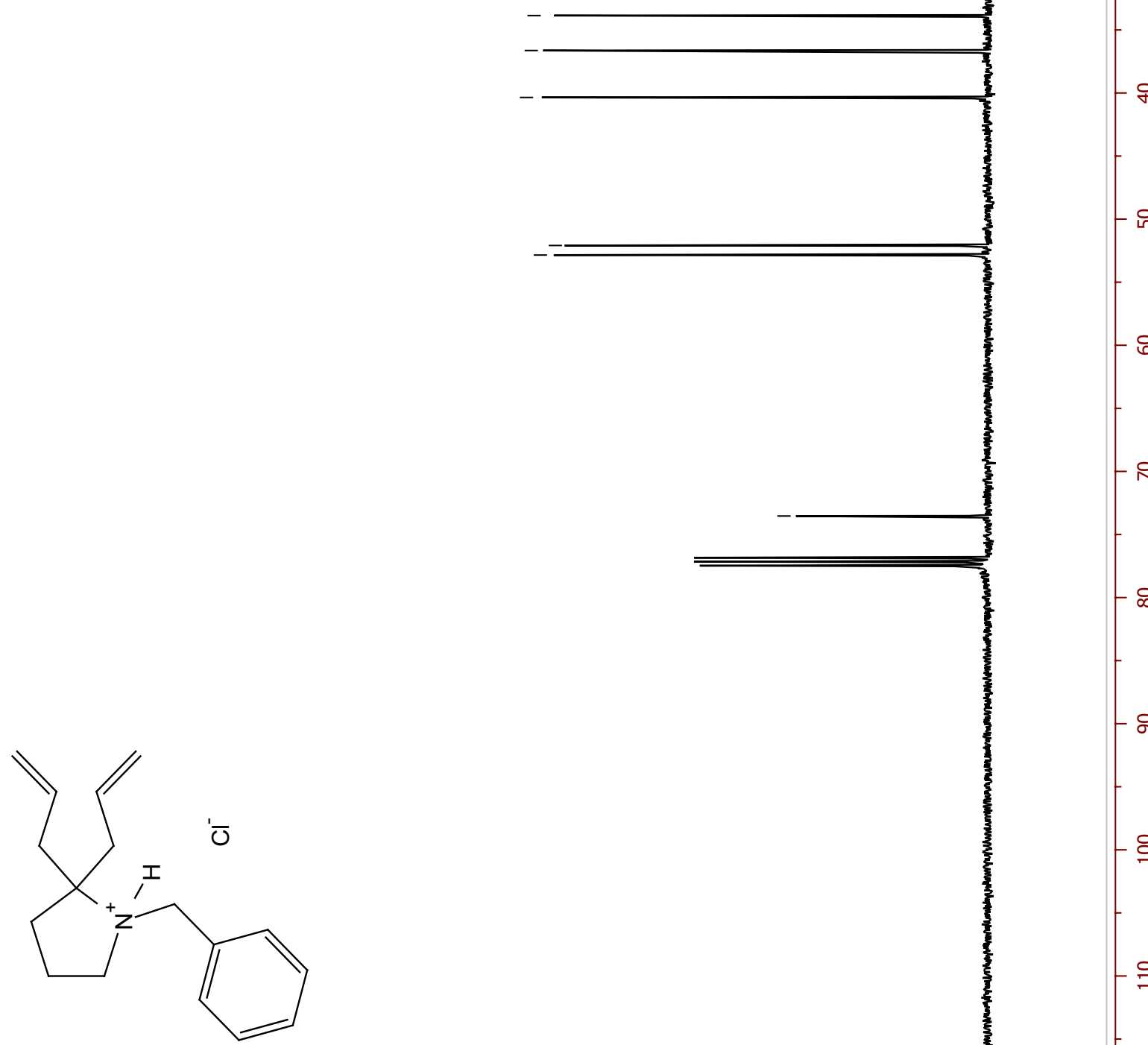

8L'TEI]

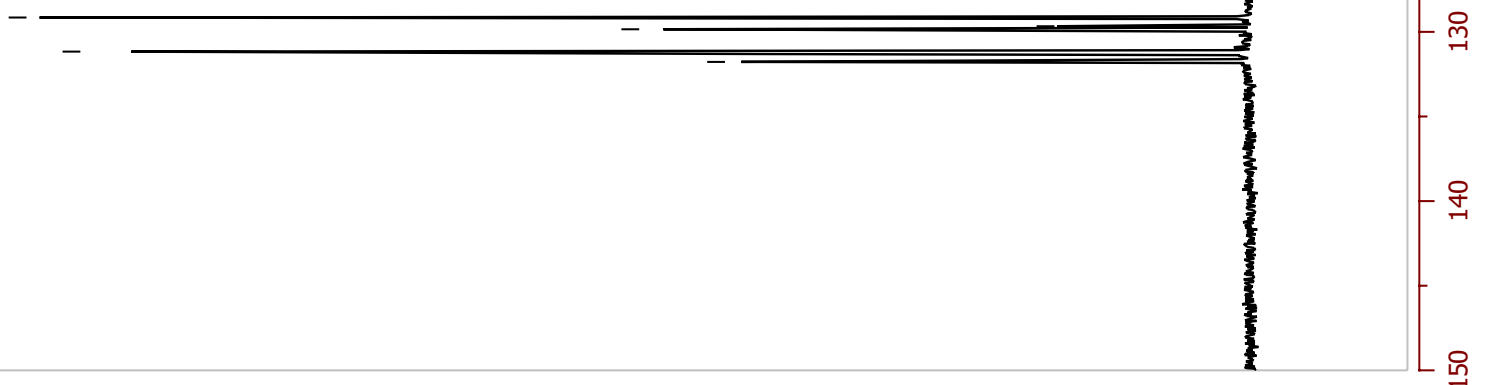


69' I-

ร8. โ-

$0 Z^{\prime} Z$

sz' 2

でてー

$I \angle Z$
$Z \angle 2$

$\left.60^{\circ} \varepsilon\right]$
II' $\varepsilon$ ]

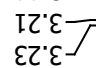

$\left.\begin{array}{l}\left\lfloor 8^{\prime} \varepsilon\right. \\ \varepsilon 8^{\prime} \varepsilon \\ t 8^{\prime} \varepsilon- \\ \angle 8^{\prime} \varepsilon\end{array}\right]$

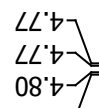

[8. 6 .

SZ'S

IE's $\frac{\bar{F}}{\bar{F}}$

$\downarrow \varepsilon ' s-$

ธE' $\triangle$

$\varepsilon 6$ '

86.5

86 '5-

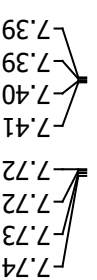

6L'II-
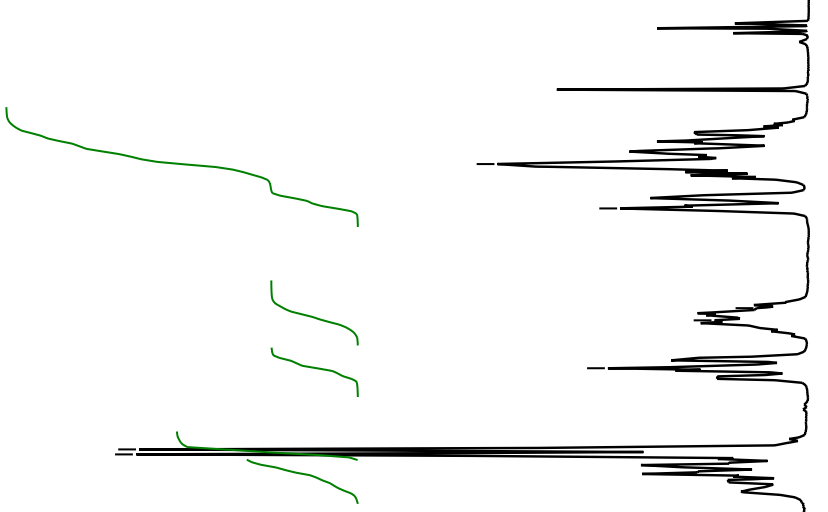

F 012
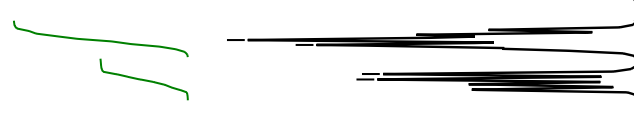

I- $20^{\circ} \mathrm{z}$

$-m$
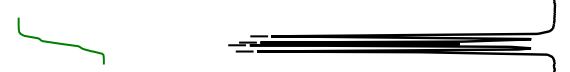

$66^{\circ} 0$

$\checkmark$

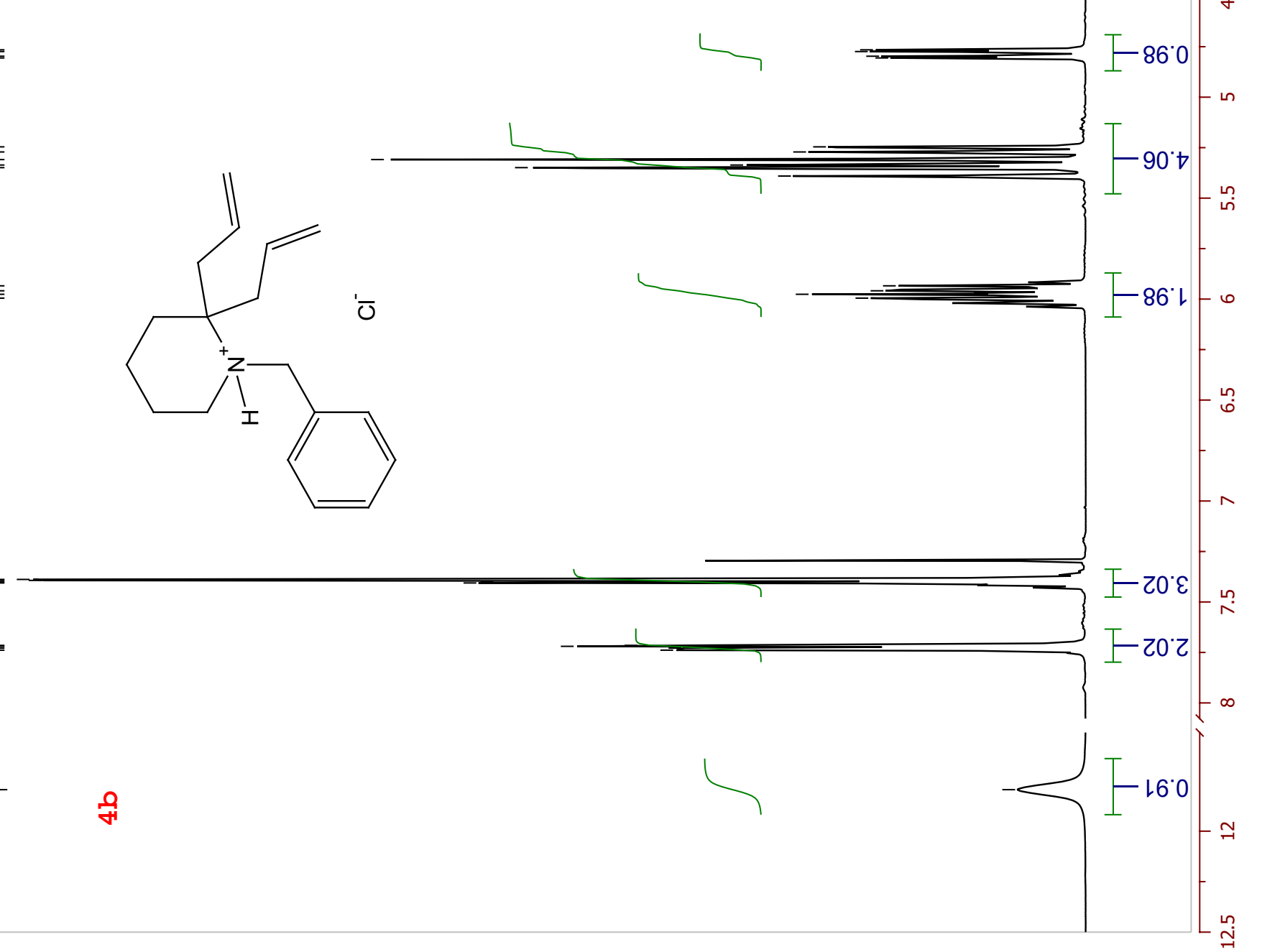


$00.81-$

SS'IZ-

$\angle \varepsilon^{\prime} I \varepsilon-$

$6 S^{\circ} \downarrow \varepsilon-$

$\downarrow 8^{\circ} 0 b-$

os' 9 t -

9६'६૬-

I0 $\angle 9-$

f

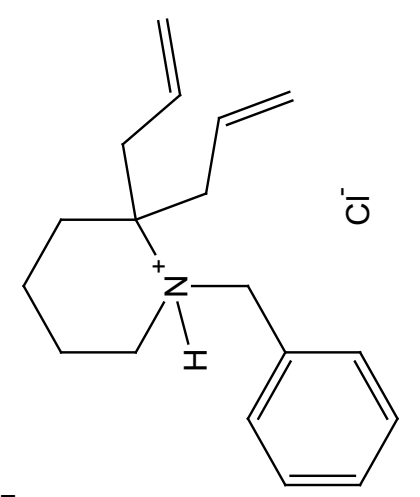

08.0ZI
$00^{\circ}$ 'LI

$\angle 66^{\circ} 8 \mathrm{~L}$

2I'62I]

28.6ZI-

$\angle 8^{\circ} 0 \varepsilon \mathrm{I}^{-}$

$\angle 9^{\circ}$ 'TEI

$86^{\circ}$ IEI

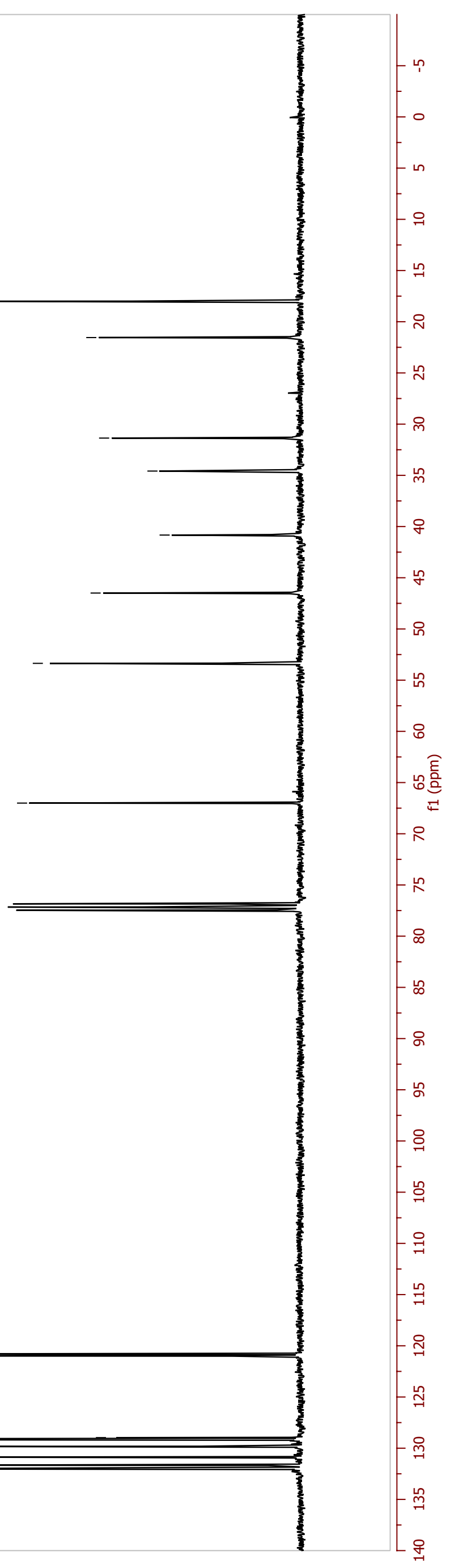


$\frac{00^{\circ} 0^{-}}{20^{\circ} 0^{\prime}}$

$6 Z^{\prime} T_{1}$

O६' $[$ ]

It I

$\varepsilon b^{\prime}[-$

89. IT

IL' I

$9 z^{\prime} Z_{7}$

$8 Z^{\prime} Z$

०६' $ح-$

乙䬺

โ沱

$\varepsilon t^{\prime} z$

st'

$\varepsilon 9 \cdot ح$

โร ${ }^{\circ} \varepsilon-$

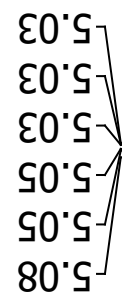

$26^{\circ} \mathrm{S}$

$76{ }^{\circ} \mathrm{s}$

$76^{\circ} \mathrm{S}^{-}$

96. $\mathrm{s}$

96. 9

$86^{\circ} \mathrm{s}$

8' $\mathrm{T}$

$0 Z^{\circ} \angle$

ZZ' $\angle$

$\left.\varepsilon Z^{\prime} L\right]$

$\angle Z^{\circ} \angle T$

$6 Z^{\circ} L$

IE' $L$

$6 \varepsilon^{\prime} L$

It $L^{\circ}$

$\underset{7}{0}$
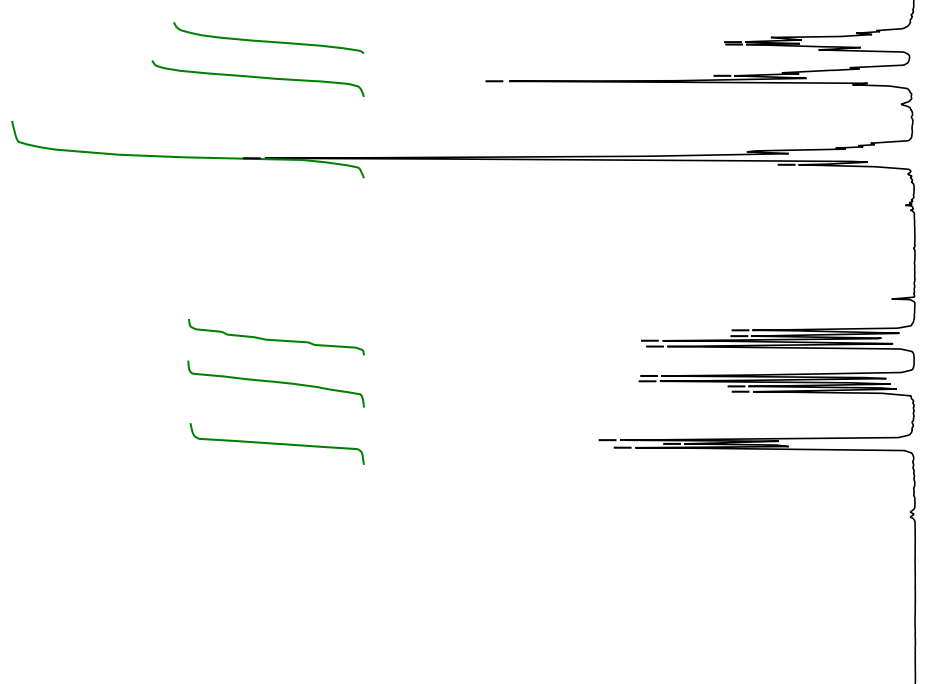

F $2 \nearrow^{\prime}$

F-87'

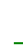

-てレナ

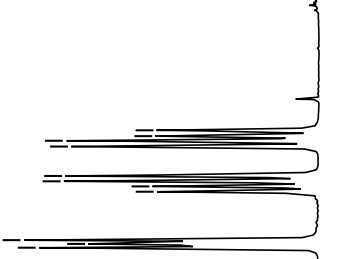

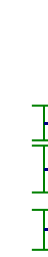

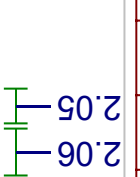

$-90^{\circ} \mathrm{\sim}$

F $\varepsilon 0^{\circ} z$

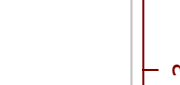

3

F $10^{\circ} \mathrm{z}$

$\stackrel{\stackrel{n}{m}}{m}$

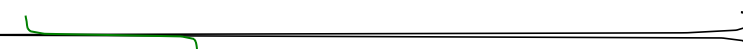

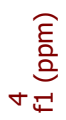

$\stackrel{?}{+}$

F 90 七

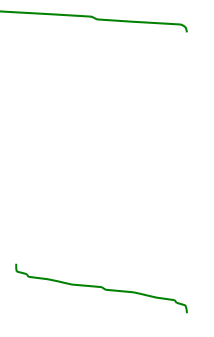

$-90^{\circ} \mathrm{t}$

.

?
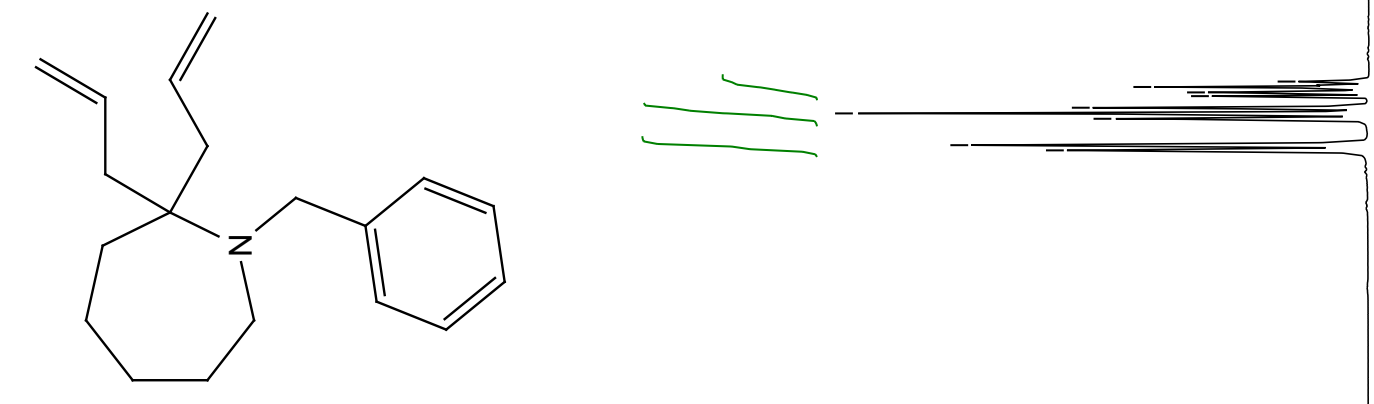
$\neg I^{\circ} 0 \varepsilon$ 乙

$\dashv 0^{\circ} 乙 \varepsilon-$

$\varsigma \varepsilon^{\prime} \angle \mathcal{E}-$

$00^{\prime} \varepsilon \vdash-$

$68^{\prime} 8 b-$

$\angle 6^{\circ} \circ S-$

ऽE' T9-

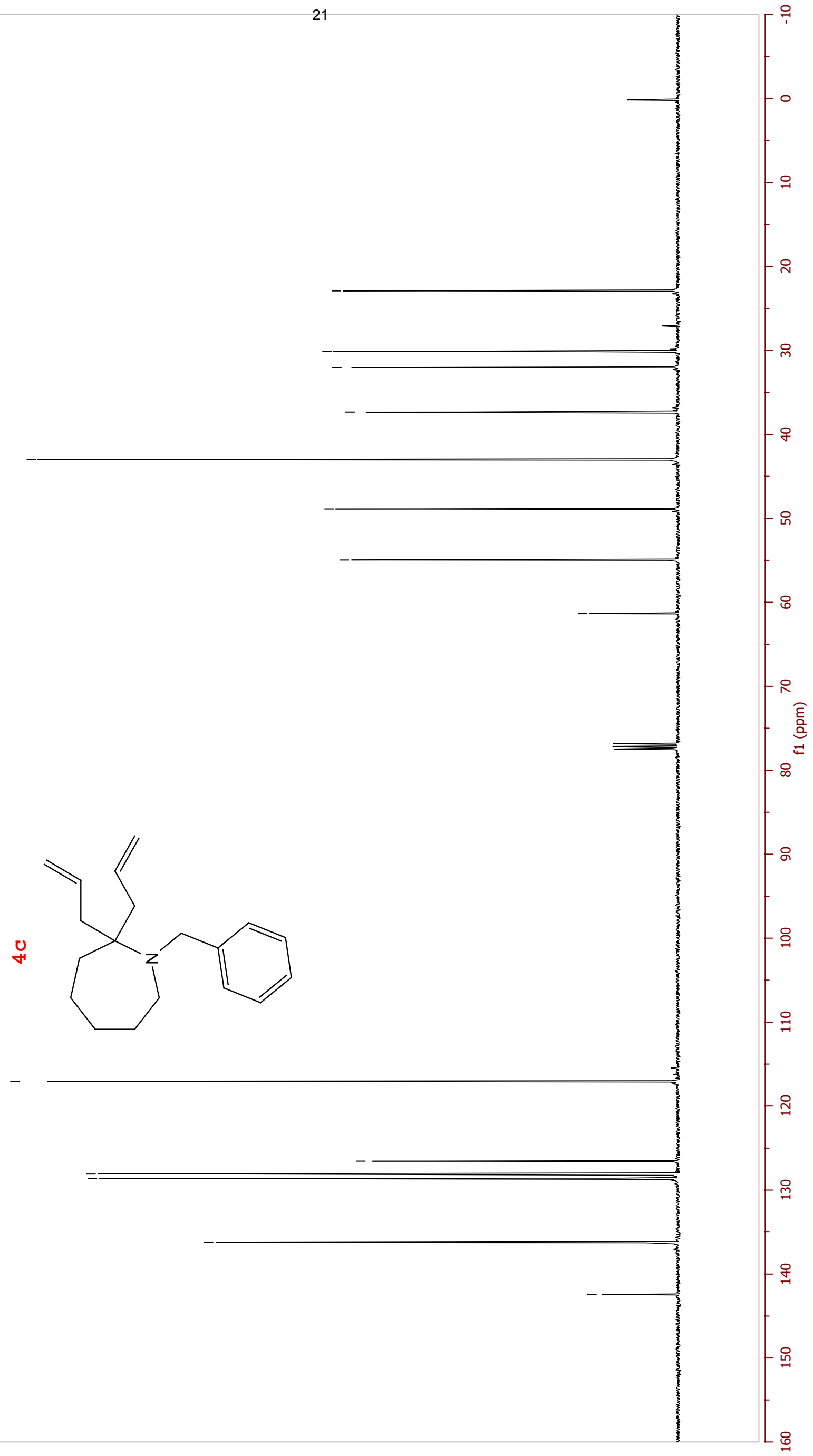

$\succ 0^{\circ} \angle I I-$

SS.9ZI 0I'8ZI

[9.8ZI

SZ'9عI-

とヤてちIー 
IL'I-

เ๐

so' $\mathrm{Z}$

$\angle 0^{\circ} \mathrm{C}^{\prime}$

$90^{\circ} \varepsilon$

$\varsigma \tau^{\prime} \varepsilon$

เ $\left.t^{\prime} \varepsilon\right\urcorner$

乙8'

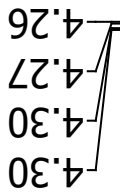

$\angle Z^{\prime} \angle$

$0 \varepsilon^{\circ} \angle$

$0 \varepsilon^{\prime} \angle$

$\succ \varepsilon^{\circ} \angle$

SE' $\angle$

$9 \varepsilon^{\prime} \angle$

$9 \varepsilon^{\circ} \angle$

$8 \varepsilon^{\circ} L$

$6 \varepsilon^{\prime} L$

It $\angle$

$09^{\circ} \mathrm{L}$

[9' $\angle$

$69^{\circ} \mathrm{L}$
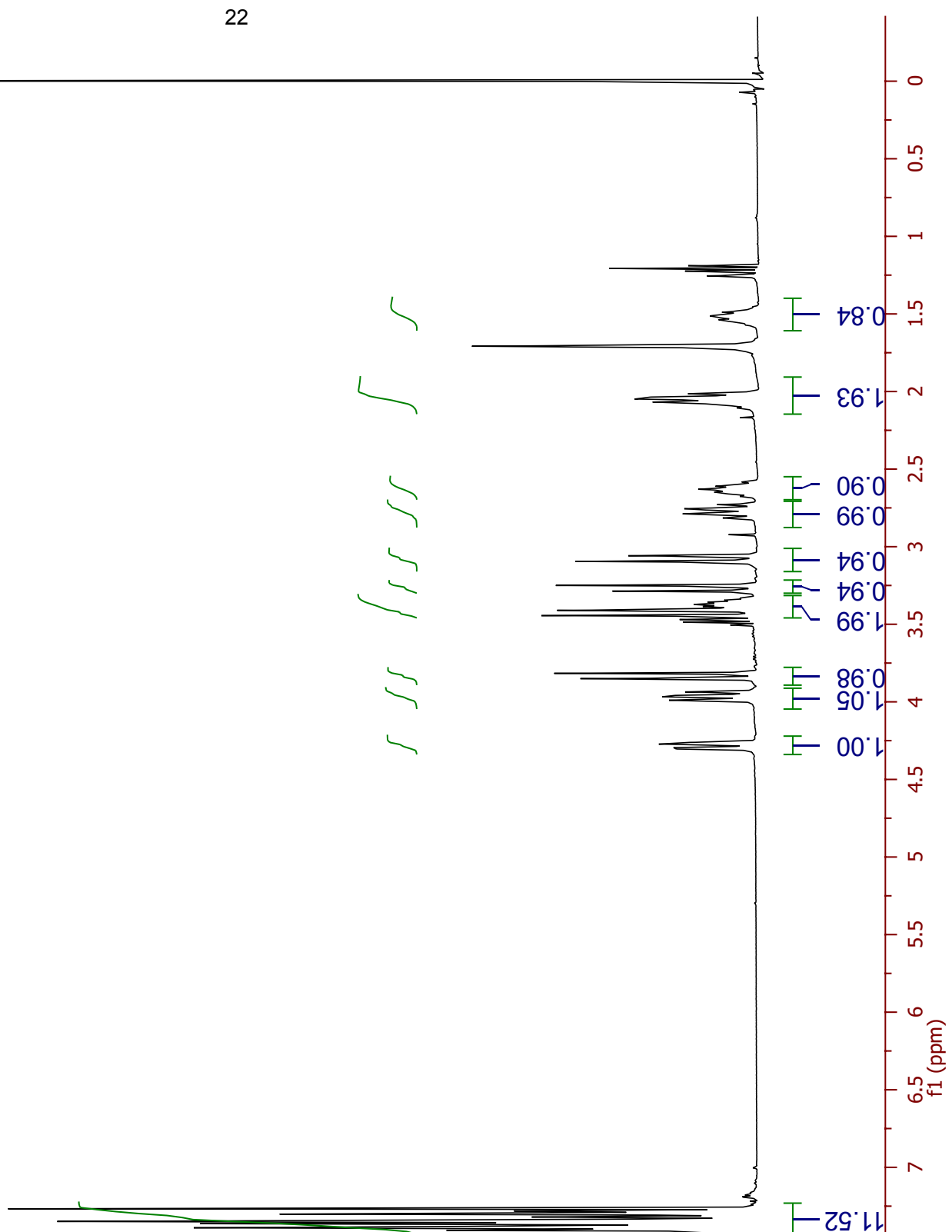

I $\varepsilon 6^{\circ}$ เ $\sim$

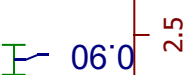

F $66^{\circ} 0$

$F+6.0^{-m}$

E $76^{\circ} 0^{\circ}$

凡 66. ᄂ

F $86^{\circ} 0$

F $90^{\circ}$.

F $00 \%$

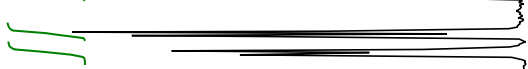

F-29'L

互 $202^{-}$

凡 10.8

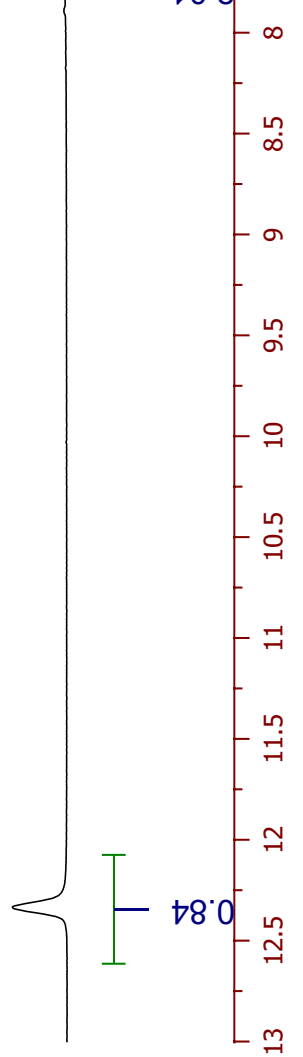


$09^{\circ} 6$ I-

$\downarrow \varepsilon \cdot \downarrow \varepsilon-$

$S t^{\circ} \angle \varepsilon-$

てとてちー

$22{ }^{\prime} 2 S_{7}$

s' $2 \varsigma^{\prime}$

SS'SL-

$6 \angle L C I$

$60{ }^{\circ} 8 \mathrm{~L}$ -

ع6.8ZI-

$\angle I ' 6 Z I$ -

हZ'6ZI]

SL'6ZI

06.6ZI

† $0 \varepsilon Г$

$\neg I^{\prime} I \varepsilon I-$

I乙 ऽEL

[9'SEI
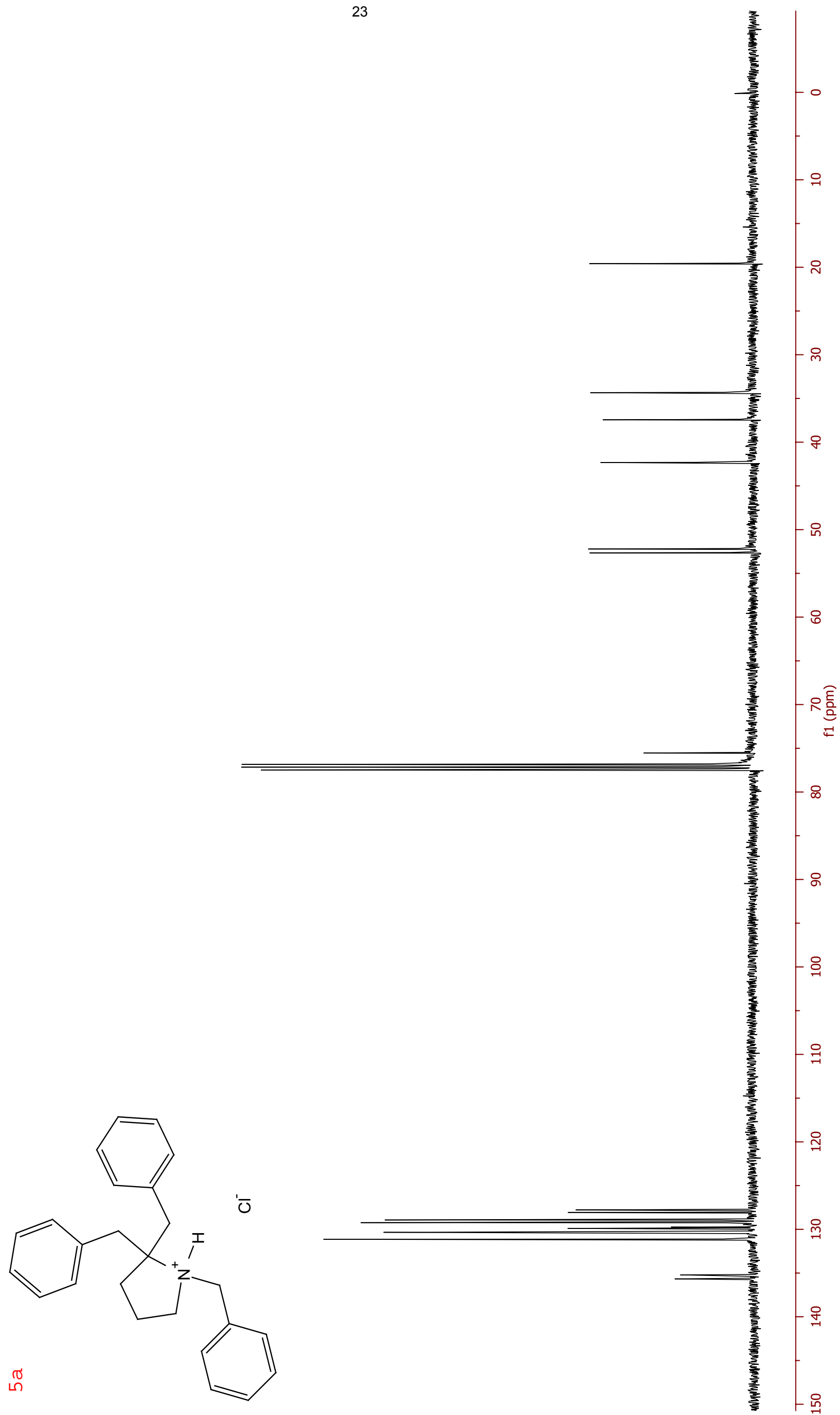
$\left.8 \varepsilon^{\prime \prime} I\right]$

OS'I-

$\angle 9^{\circ}$ I-

ZS'Z

$95^{\circ} 2 \frac{1}{1}$

$09{ }^{\circ} \mathrm{z}$

$82^{\prime} \varepsilon>$

$\varepsilon \varepsilon^{\prime} \varepsilon^{-}$

$20{ }^{\circ}$
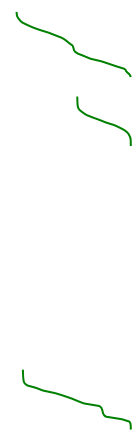

5<smiles>CC[C]1CCCCC1(Cc1ccccc1)Cc1ccccc1</smiles>

SI'L $\angle I^{\circ} \angle$ $8 \mathrm{I}^{\circ} \mathrm{L}$ $0 Z^{\prime} \angle \sqrt{ }$

IZ' $\angle$

I $Z^{\prime} L$ $\rightarrow Z \cdot L$

$S Z^{\circ} L$

$\angle Z^{\circ} \angle$

$82^{\circ} L$

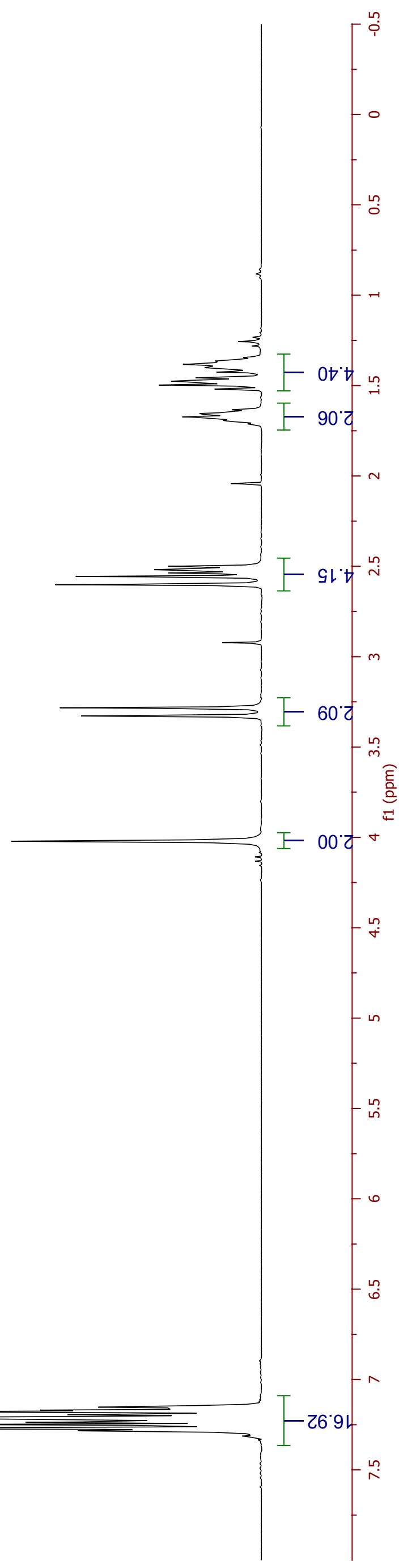


It 02

tI'SZ-

$\angle 8.62-$

$8 L^{\prime} L \varepsilon-$

t8'st-

0S'2S-

$26^{\circ} 65-$

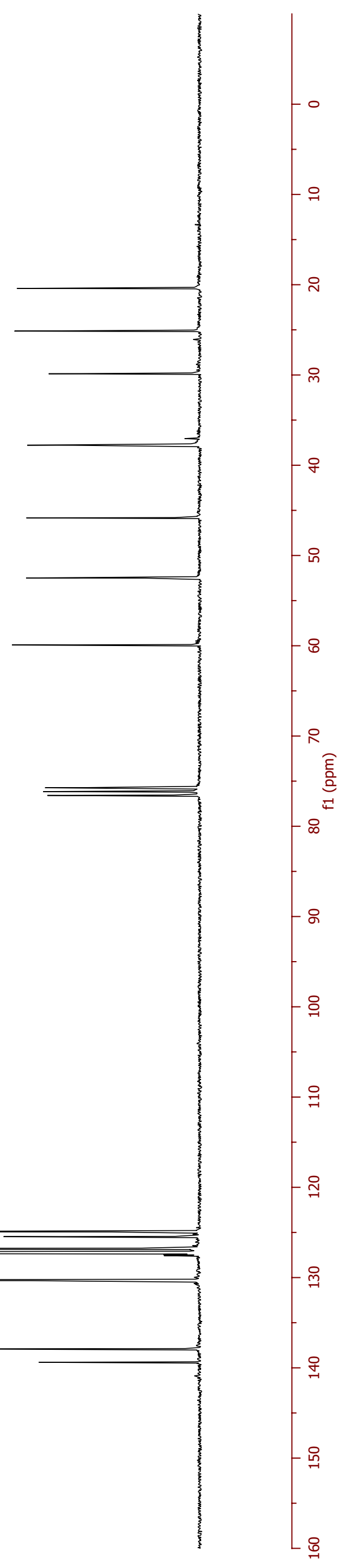

$\varepsilon 6^{\circ} \circ Z \mathrm{~T}$

$\angle t^{\circ} \mathrm{CL}$.

8L'9ZT]

9I' $\angle Z T-$

¿E' $\angle Z \mathrm{I}$

乙ย'0हा

$06^{\circ} \angle E I-$

$6 \varepsilon^{\prime} 6 \varepsilon \mathrm{IT}^{-}$

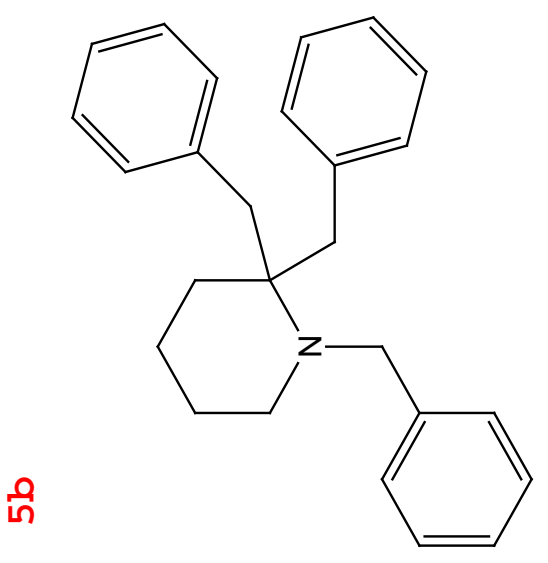




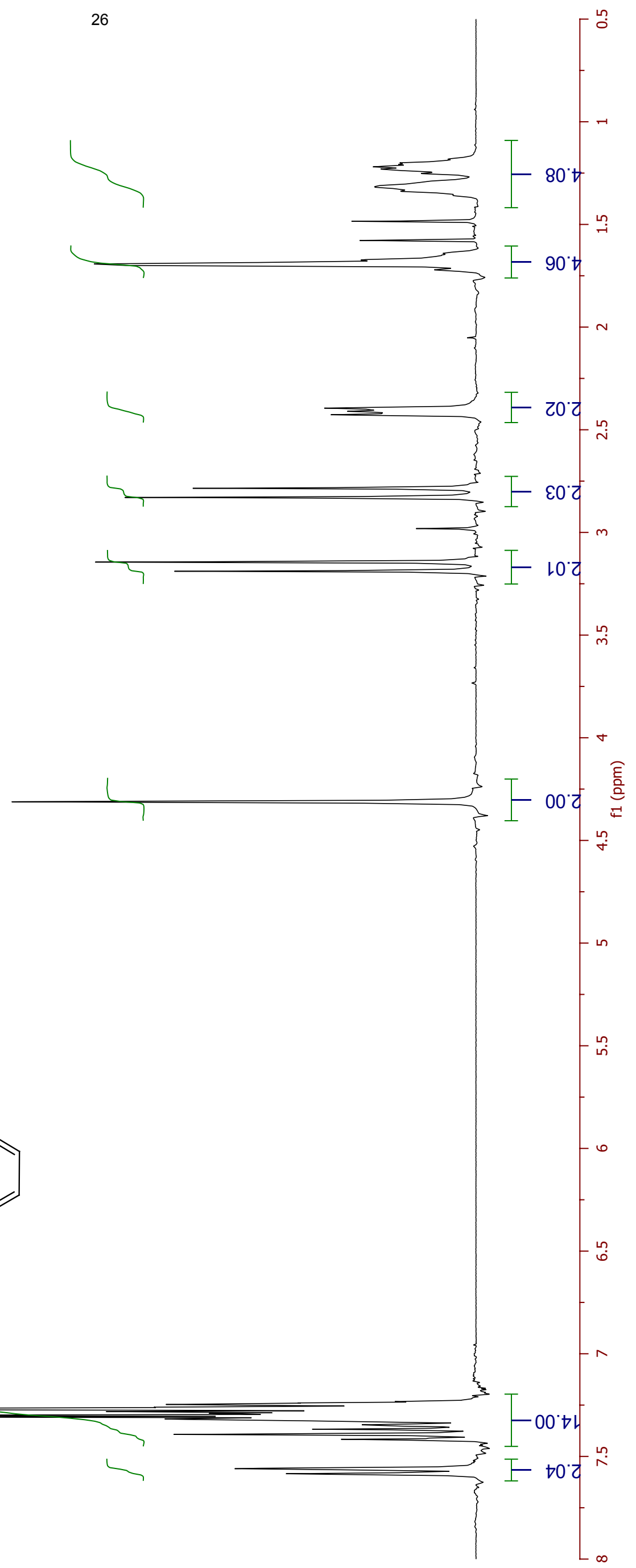


$\succ Z^{\circ} 0 \varepsilon \sim$

6เ' $2 \varepsilon-$

$00^{\circ} \varsigma \varepsilon^{-}$

68'हち-

Zt'OS-

SO'9S-

โ9'ย9-

โ0.9ZI

$09^{\circ} 9 \mathrm{ZT}$

$88^{\circ} \angle Z T$ ]

9I $8 Z T$ T

$\angle ৮$ 8ZT

$\varepsilon t^{\prime} T E I$

$6 \varepsilon^{\prime} 6 \varepsilon I-$

$\forall \angle ' Z t I-$
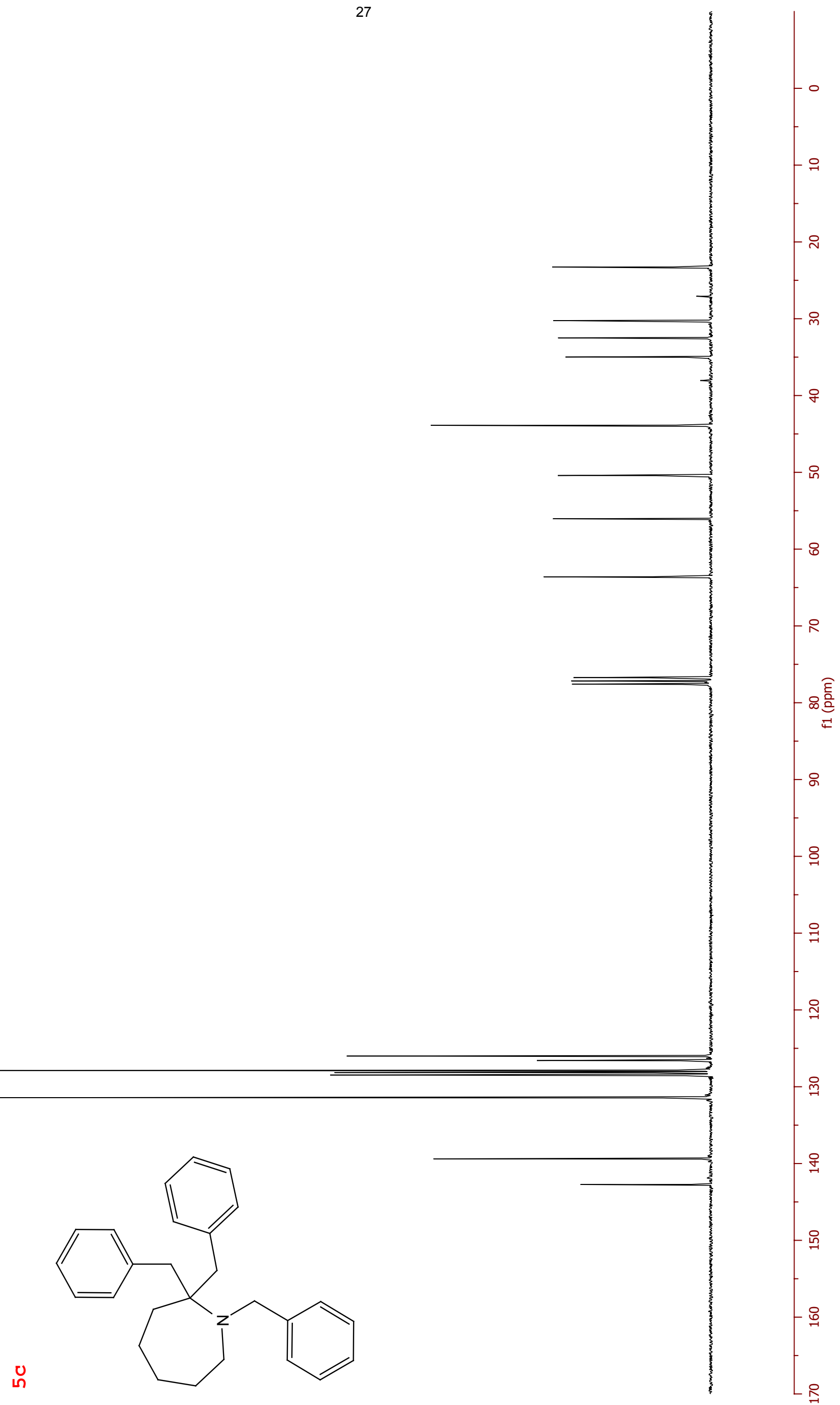


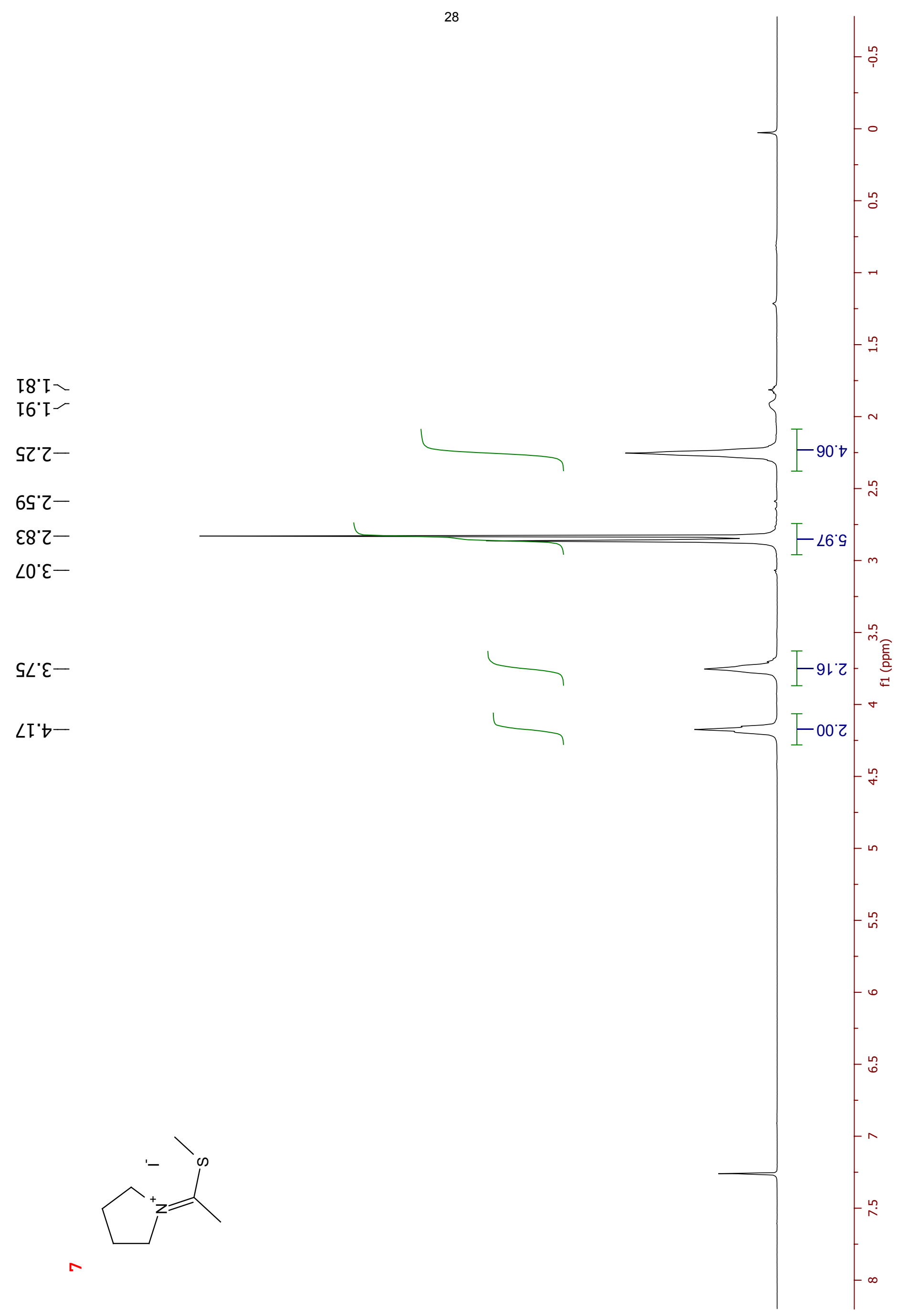


I8 $\angle I-$

20'SCT

OI'SZ'

$\angle I ' S{ }^{\prime}$

00'S ר

SI'LS

ZS' $\angle 8 \mathrm{I}-$
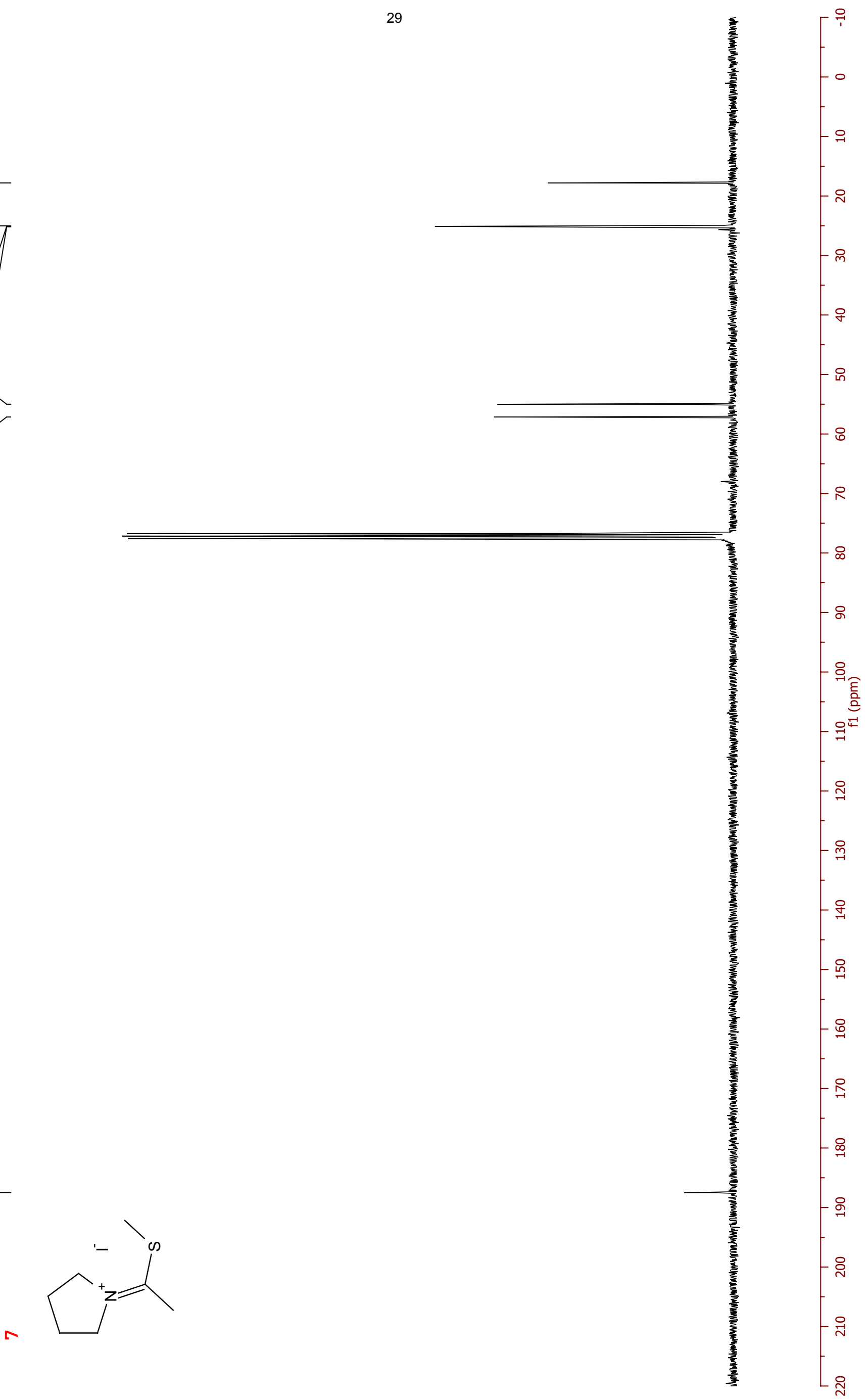


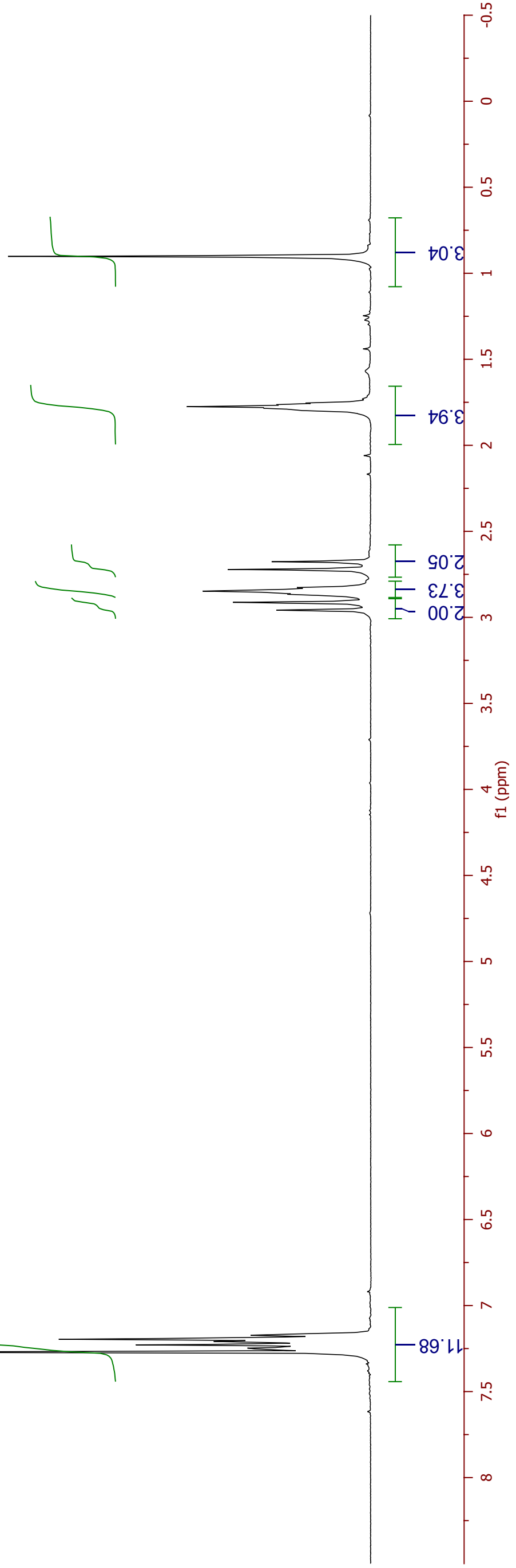

$96{ }^{\prime}{ }^{\prime}$
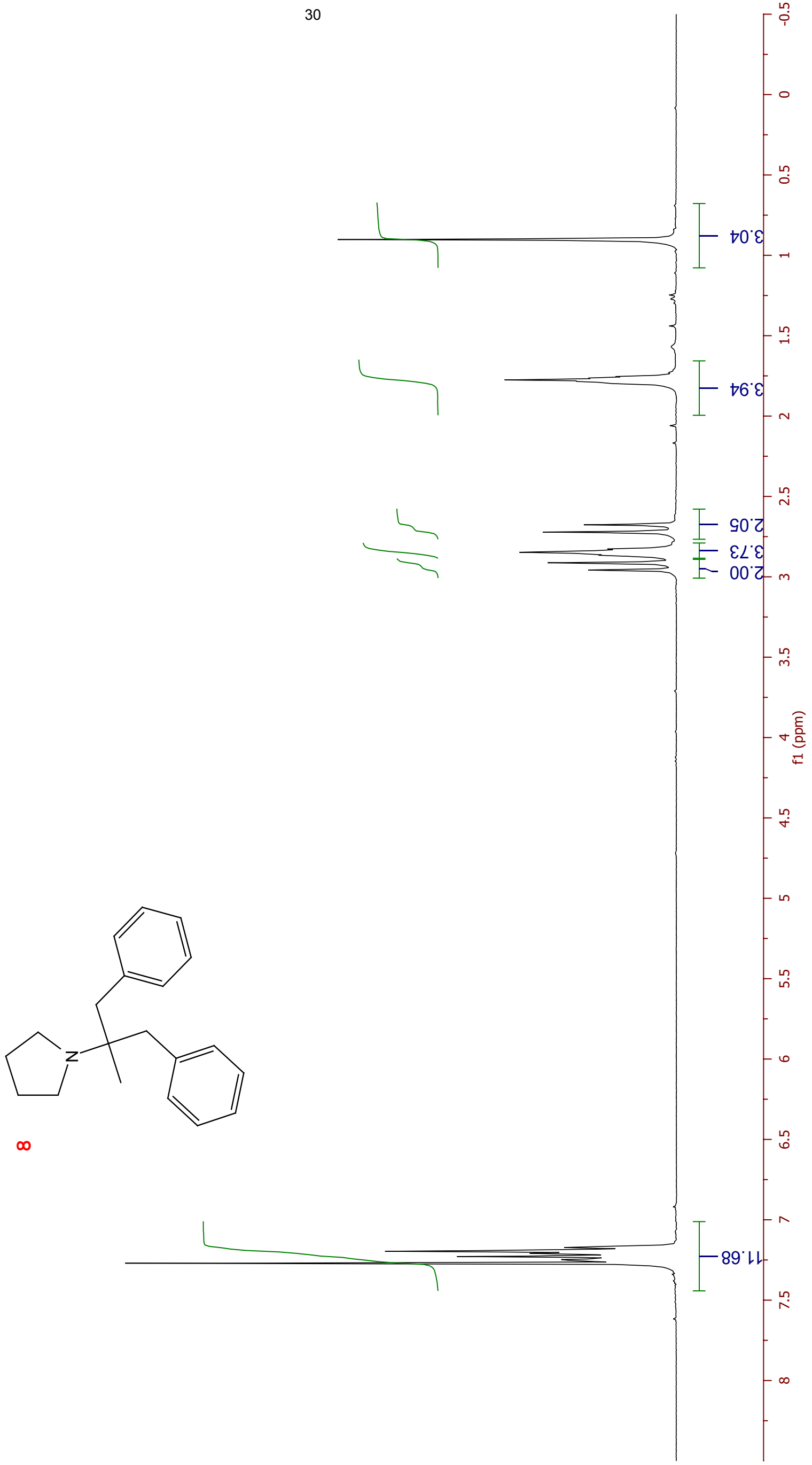

$\infty$

$0 Z$ ' $\backslash$

$\angle Z^{\prime} L^{-}$

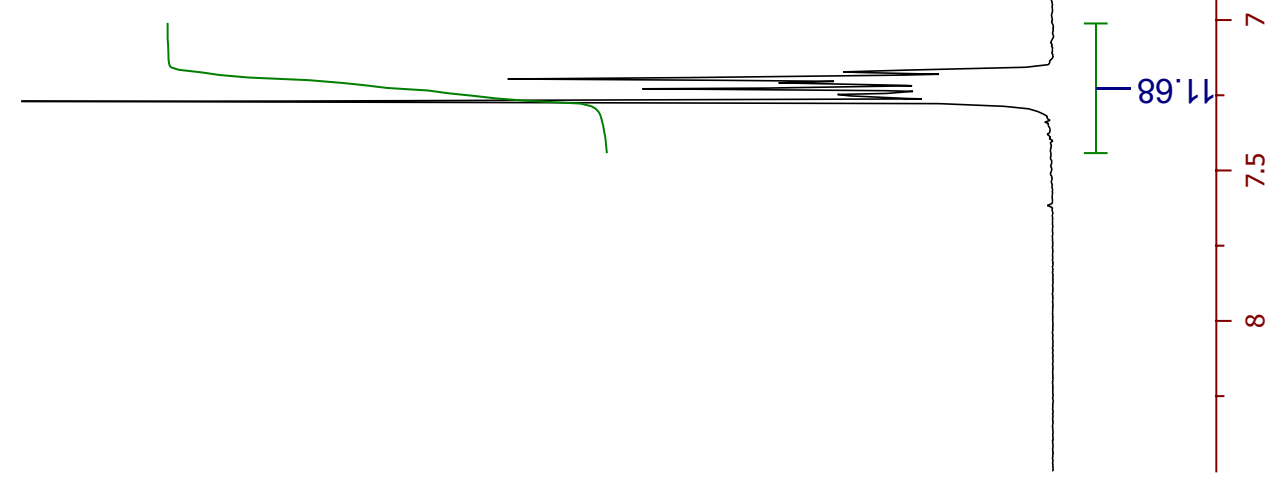


$\varepsilon 0^{\circ} \varepsilon \tau_{-}$

IL'It -

se'st-

99.85-

8L'SZI ᄂ

$9 \angle \angle Z I-$

I6.0EI-

ZS'6EI-

$\infty$

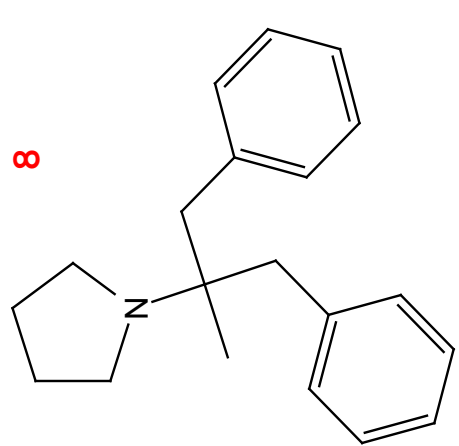




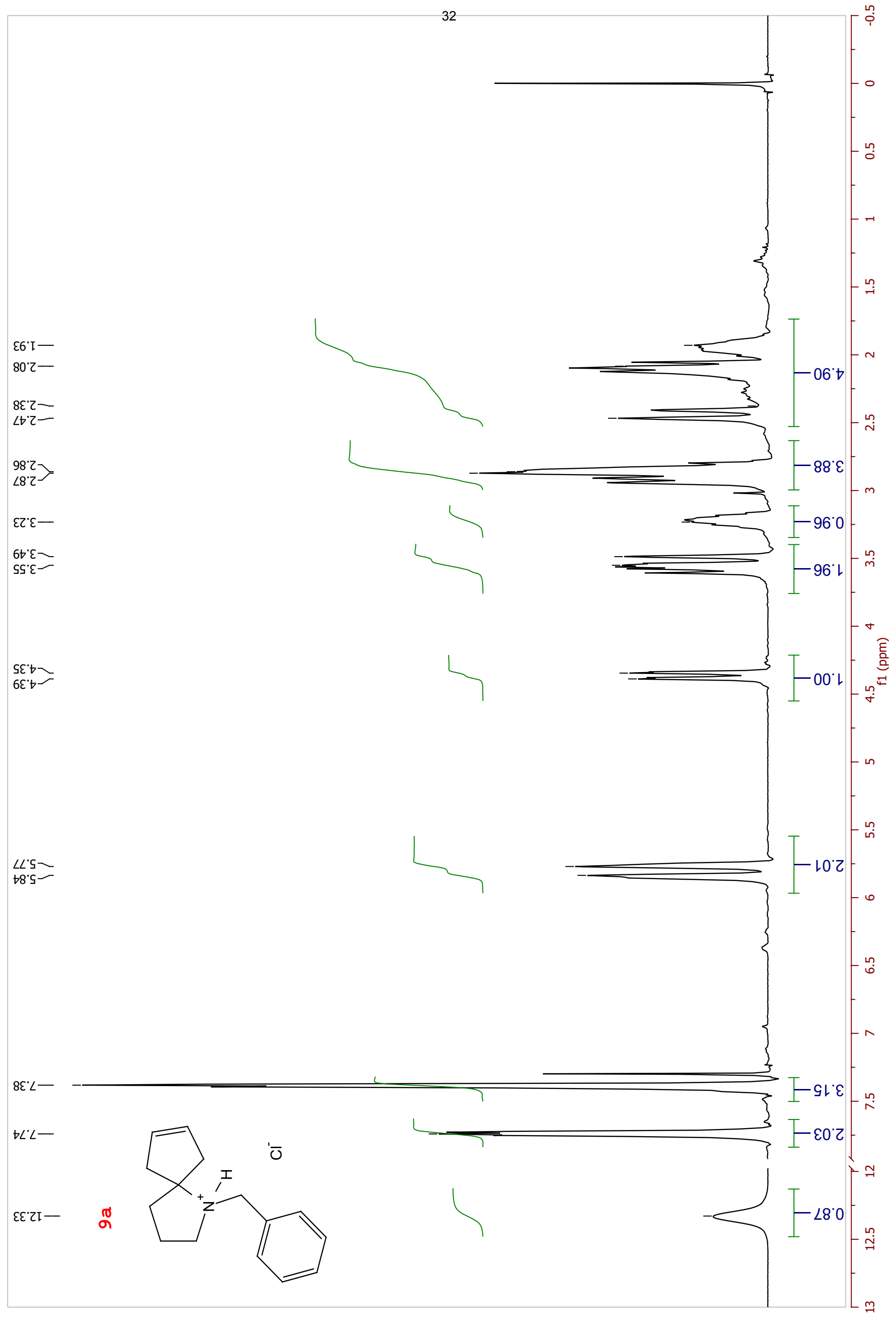


عะ'6I-

$85^{\circ} 9 \varepsilon_{\chi}$

$\angle \varepsilon^{\prime} 6 \varepsilon>$

$06^{\circ} 6 \varepsilon^{\mathcal{}}$

$\angle 66^{\circ} 8$ -

68. IS-

$26^{\circ} 9 L-$

$\angle \varepsilon^{\circ} \angle Z \mathrm{~T}$ $06.82 \mathrm{~T}$ $\angle b^{\circ} 6 Z T$ $\varepsilon \angle{ }^{\prime} 6 Z \mathrm{~T}-\bar{F}$ $\supset Z^{\circ} 0 \varepsilon \mathrm{I}$ OI'TEI
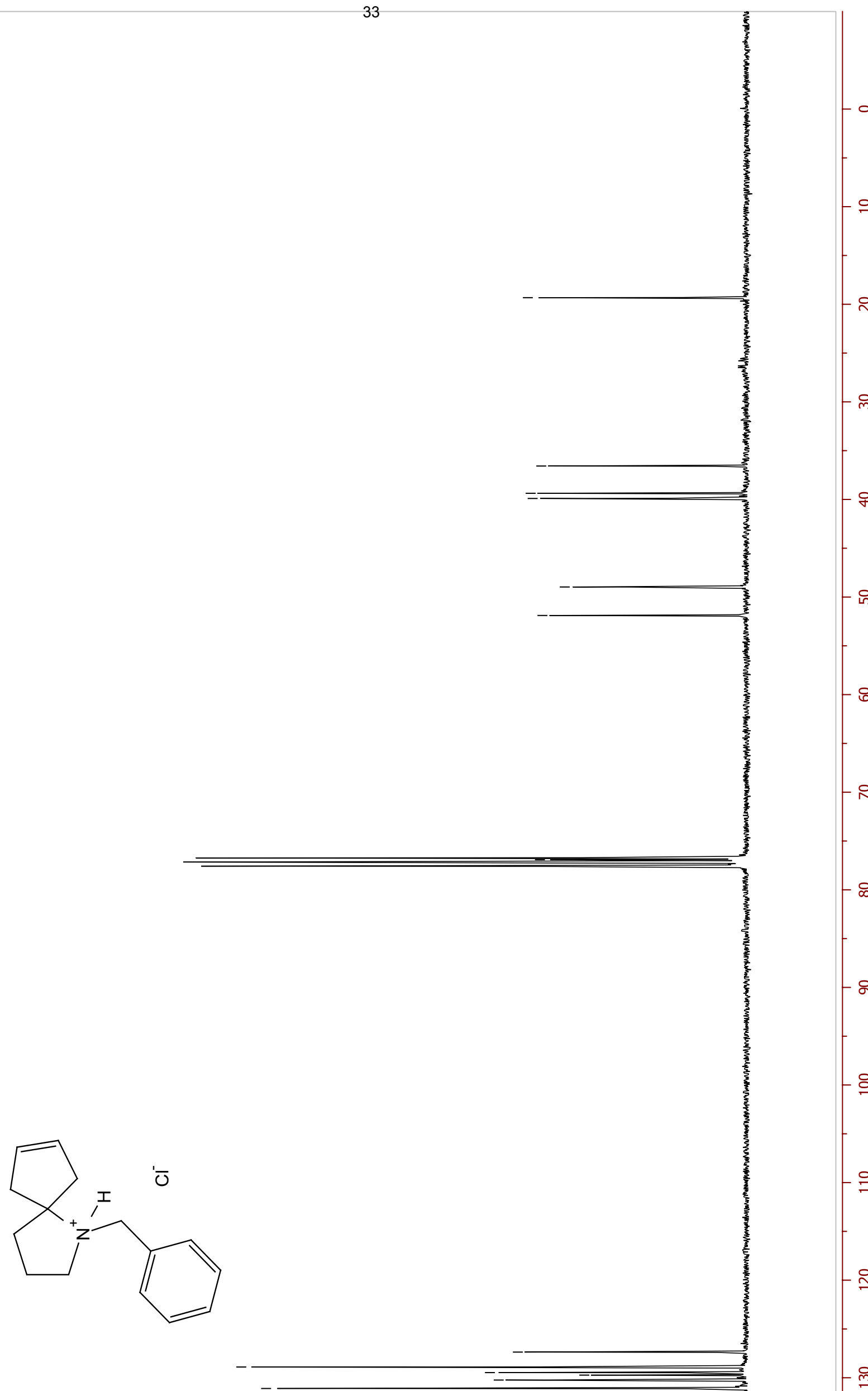
ปt. $2 \theta^{\circ}$

29.'万丁

80'て-

$\angle Z ' Z$

$0 S^{\prime} Z-$
S.

$\varepsilon \nearrow^{\prime} \varepsilon-$

8S.S-

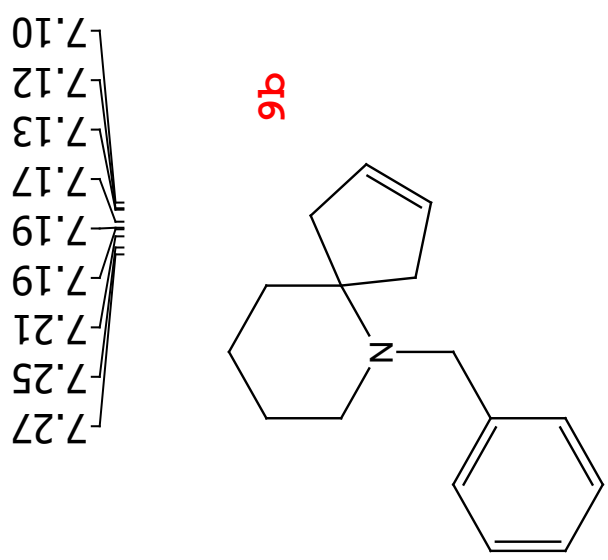

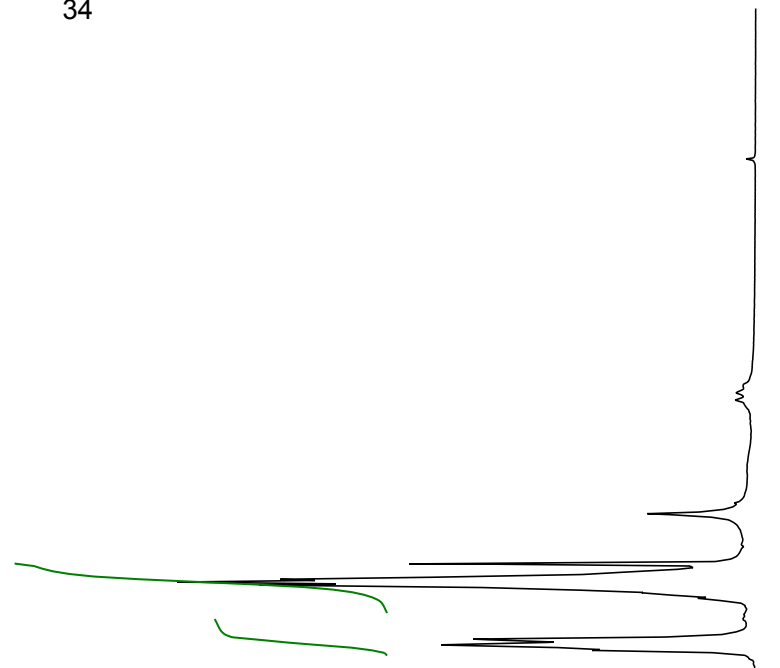

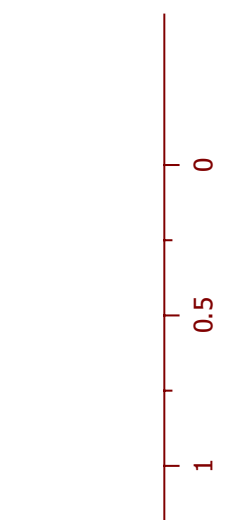

F $L \nearrow^{\circ} \nabla$
$\bar{F} 80^{\circ} 乙$

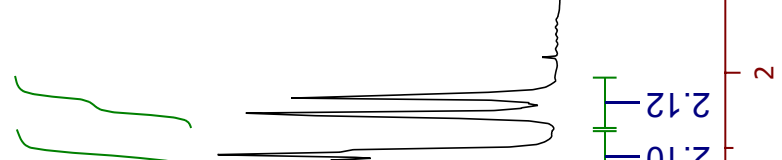

For
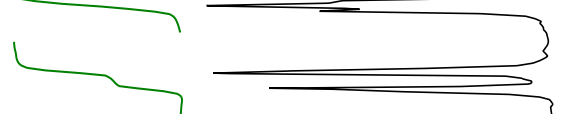

$[-002-\stackrel{n}{N}$
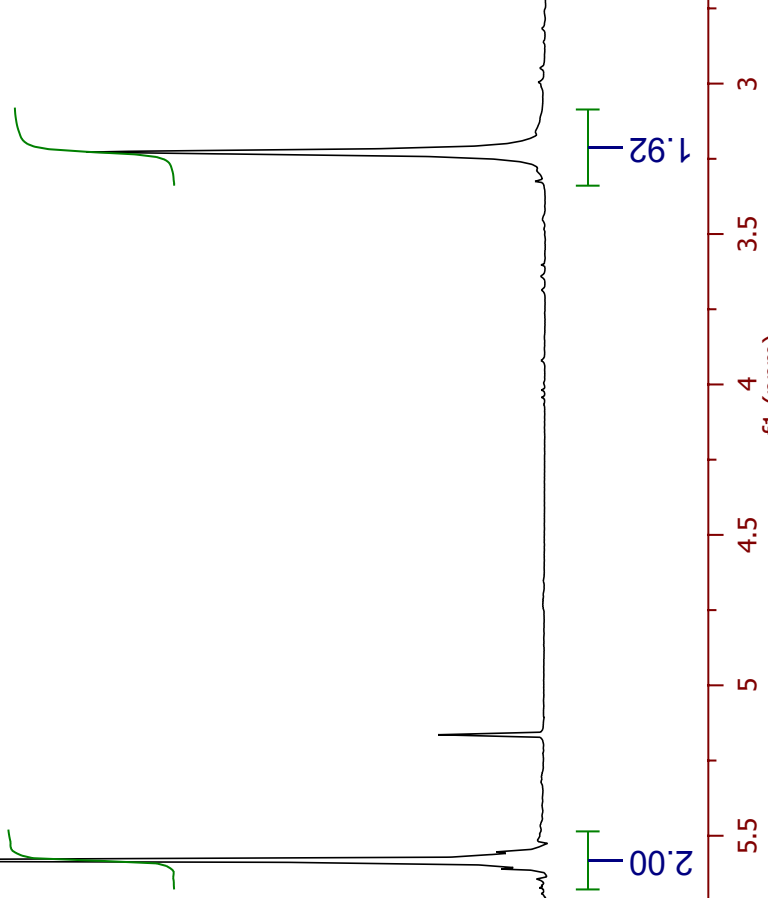

占

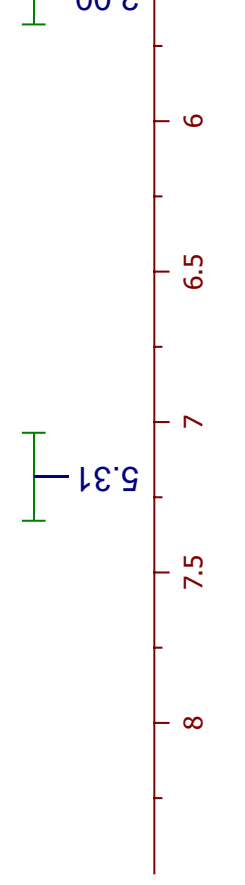


$\angle I^{\prime} Z Z-$

$2+92-$

$\angle 9^{\circ} 0 b-$

$8 \varepsilon^{\prime} 8 t-$

$69^{\circ} \mathrm{S}-$

SZ' S9-

St.9ZI SI $8 \mathrm{ZT}$

$\angle D^{\circ} 82 \mathrm{I}-$

ลำ
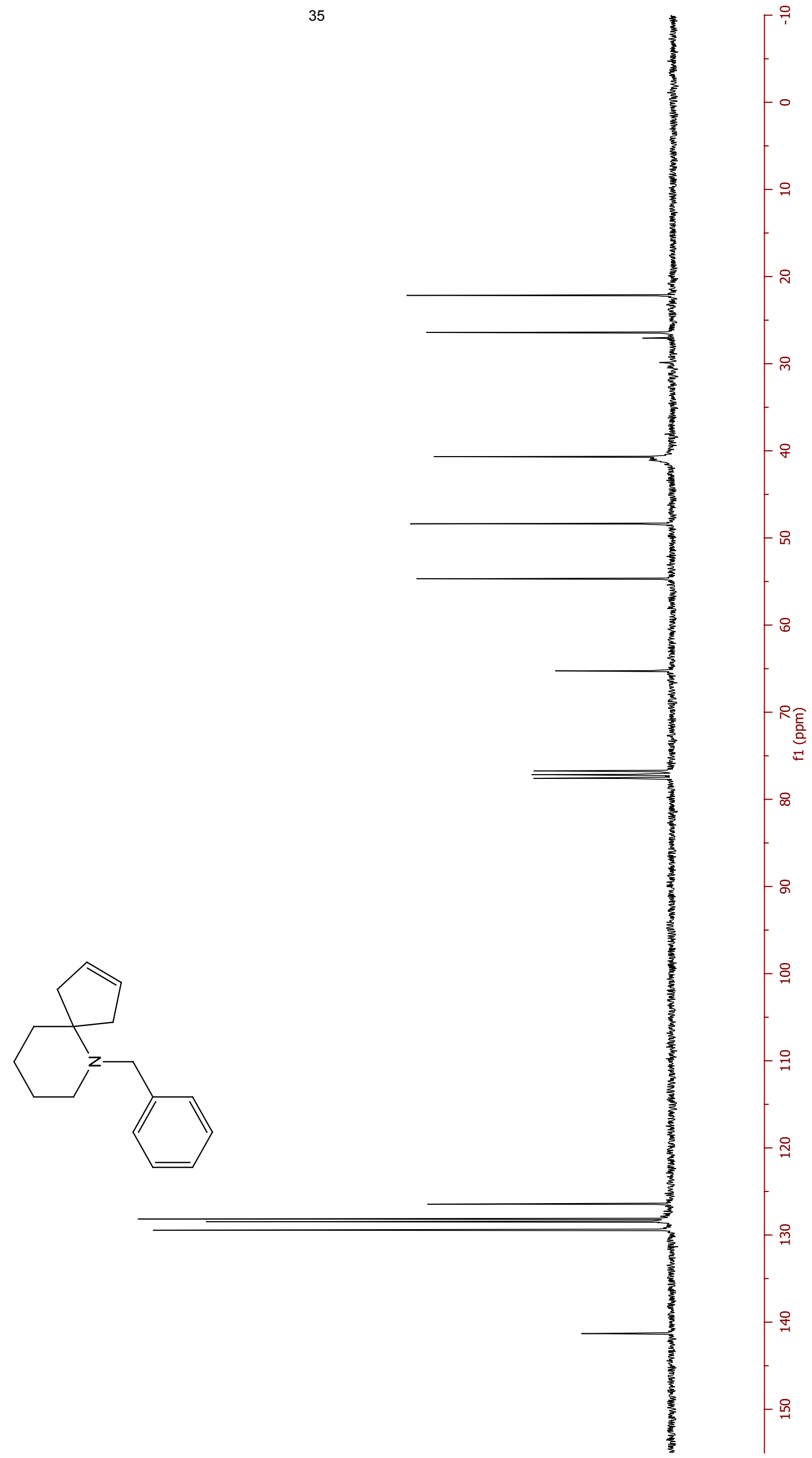

IE'ItI-

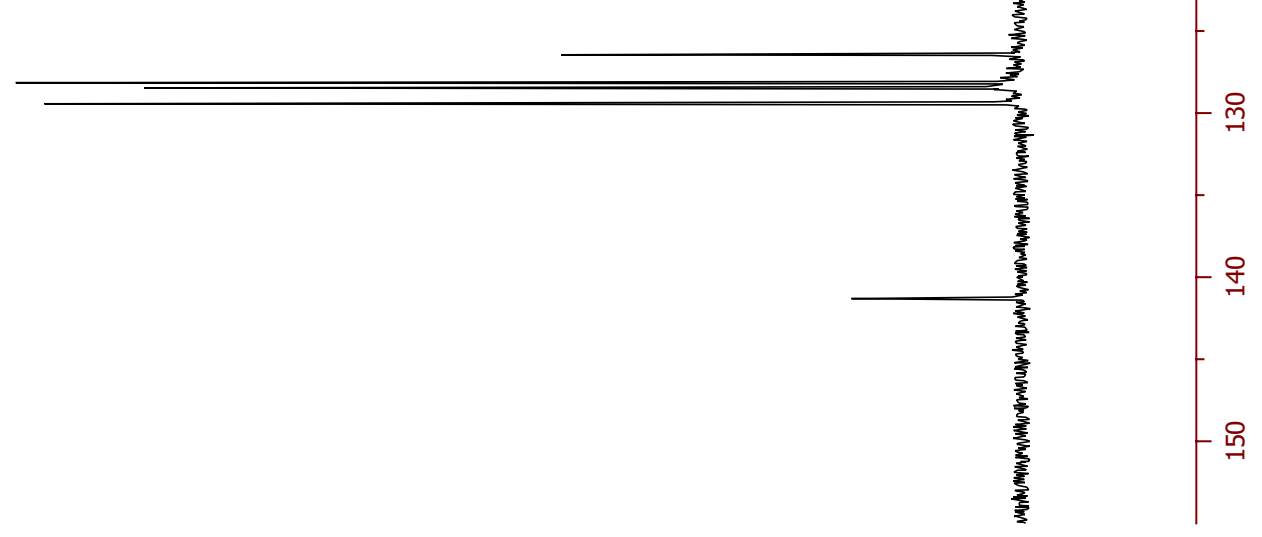


$9 \varepsilon^{\prime} \mathrm{I}$

9t' I-

ह9. I-

$\mathrm{IL}^{\prime} \mathrm{I}^{\top}$

$\left.\varepsilon I^{\prime} Z\right]$

$8 \mathrm{I}^{\circ} \mathrm{Z}-$

$66^{\circ} 2-$
$65^{\circ} 2$

乙 $t^{\prime} \varepsilon-$

$8 S^{\circ} \mathrm{S}-$

ั

$\varepsilon I^{\circ} L$

6I' $\angle$ ]

$2 Z$ 'L

$b Z^{\circ} L^{\prime}$

$\varepsilon \varepsilon^{\circ} L^{\prime}$

SE' $L$
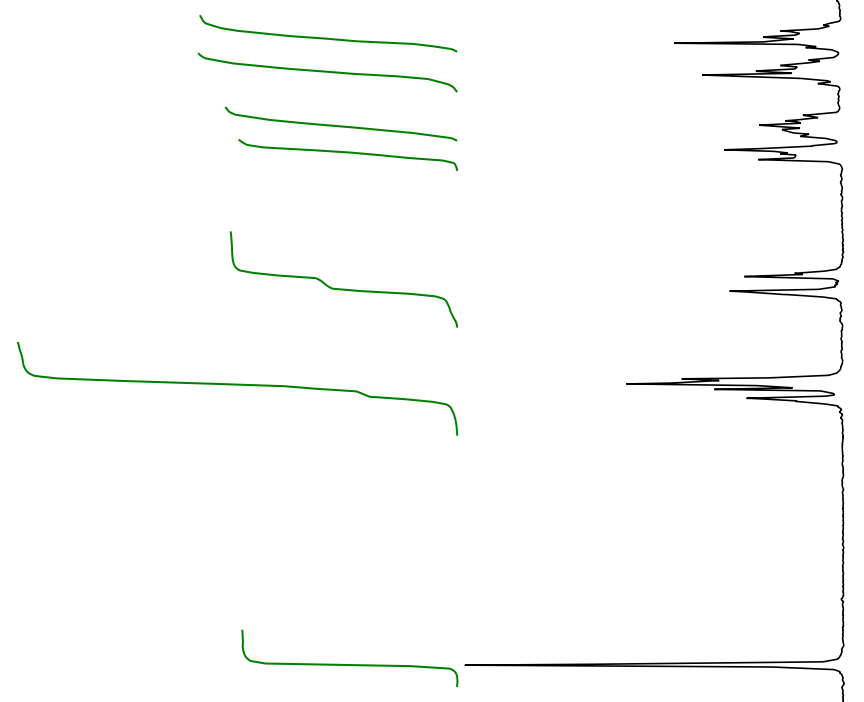

F +02

$\stackrel{n}{n}$

骨

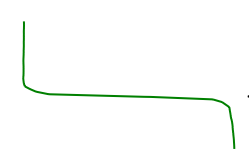

$-00 z$ 员 
OI'EZ-

$\varepsilon 8^{\circ} 62-$

$8 \varepsilon^{\prime}$ 'น

s6. $\varepsilon b$

$\varepsilon[" t b-$

$8 b^{\prime} 8 t-$

I9'SS-

E†'89-
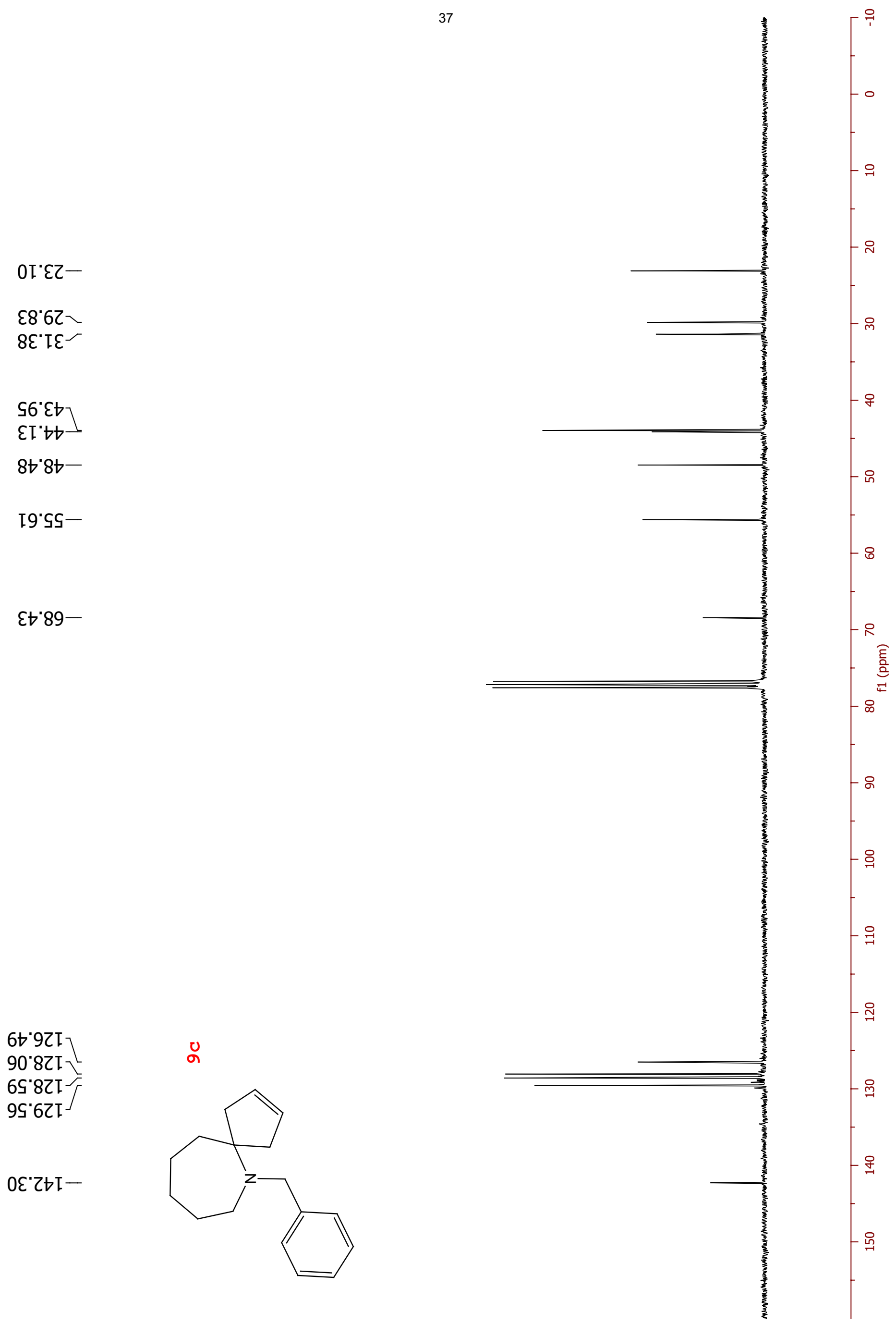
60" $I-$

89. I

$69^{\circ} \mathrm{I}$

$19^{\prime} Z$
$29^{\circ} 2^{\prime}$

IS' $\varepsilon-$

$9 C^{\circ} L$
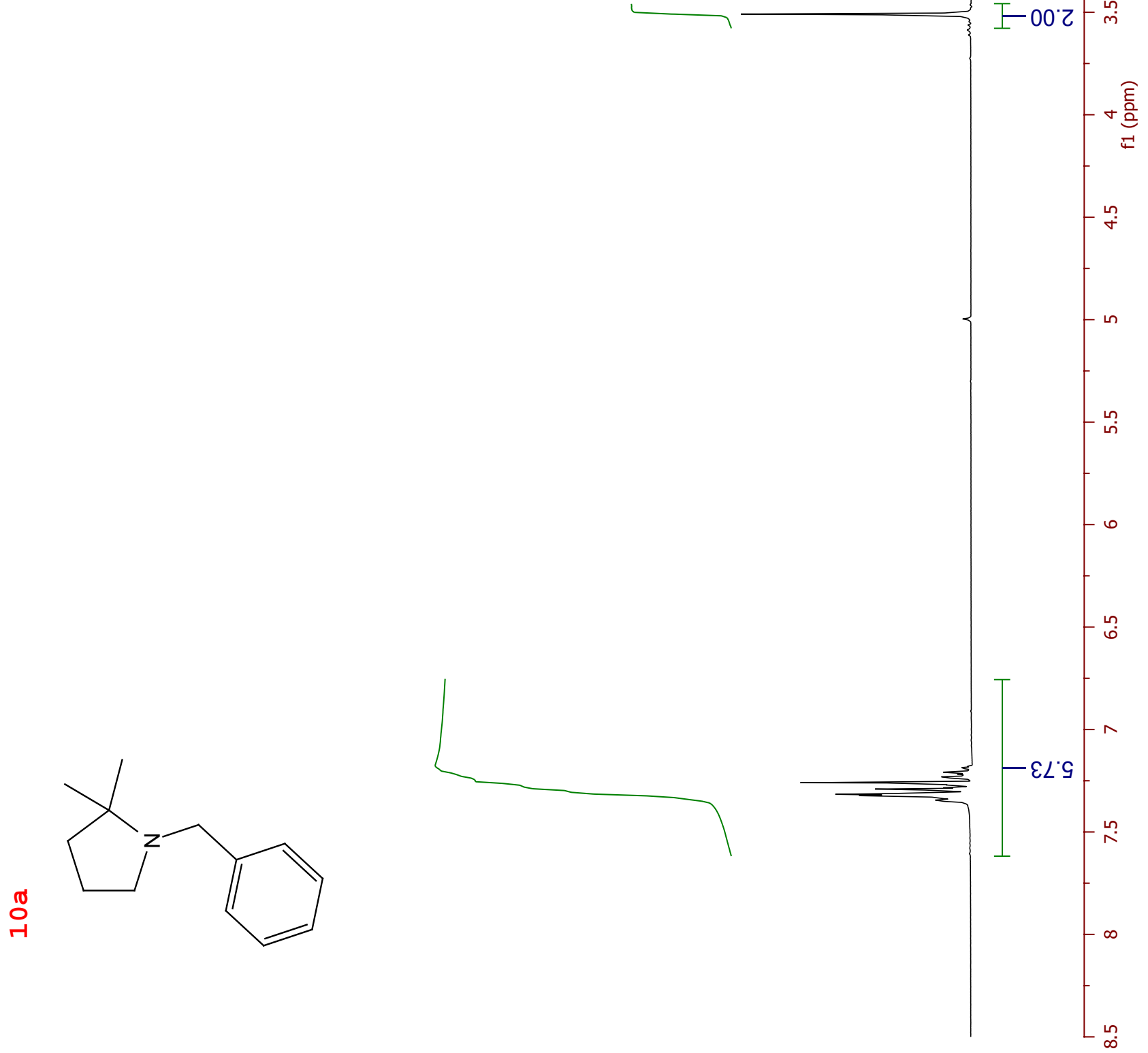
†9.0Z -

OZ'EZ-

ZI'Ob-

LO'IS -

†ह'हS-

6I'09-

\&9'9ZI

92'8ZI

$0 \angle 8 Z$ I

$\varepsilon Z^{\prime}$ ItI-
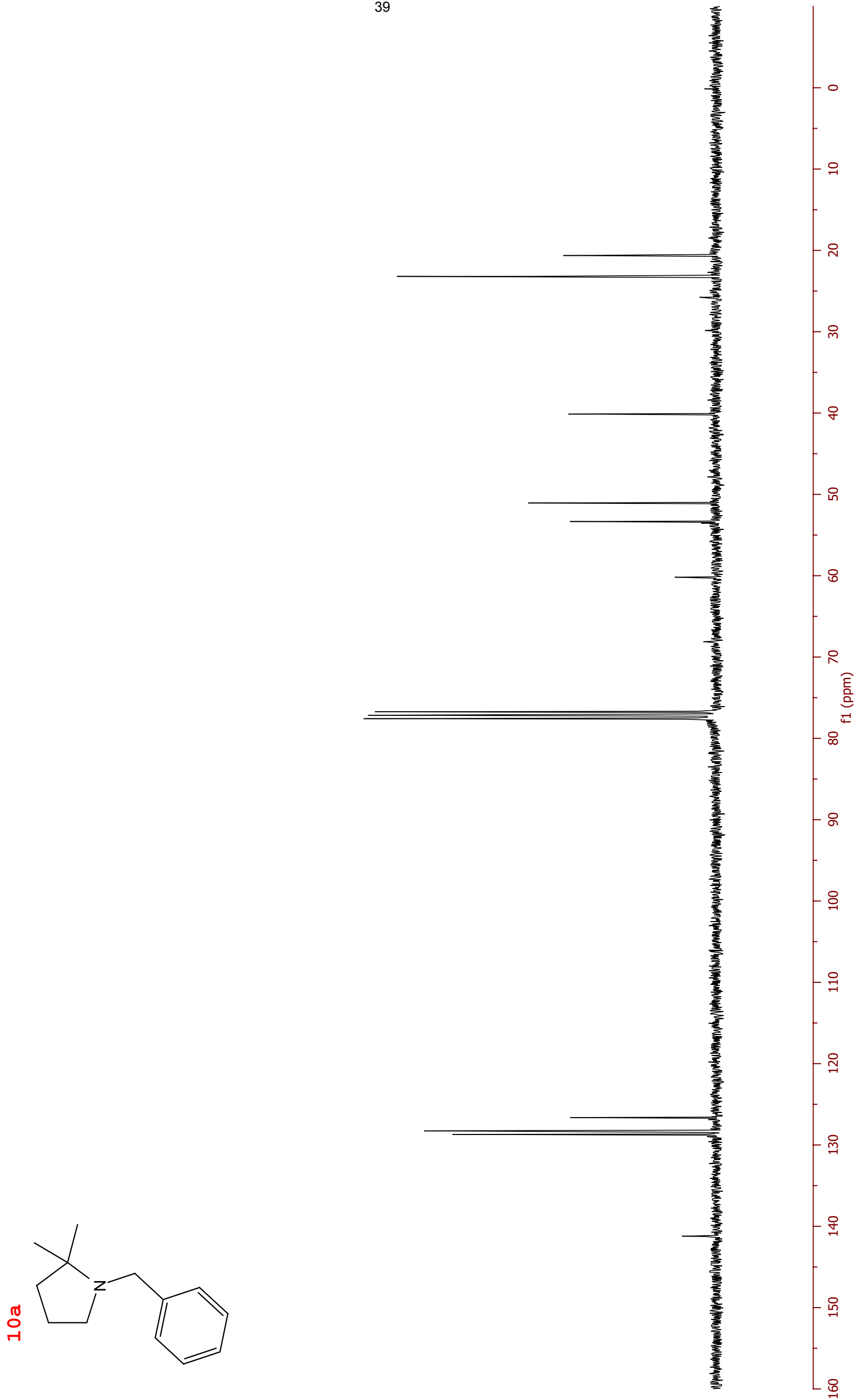


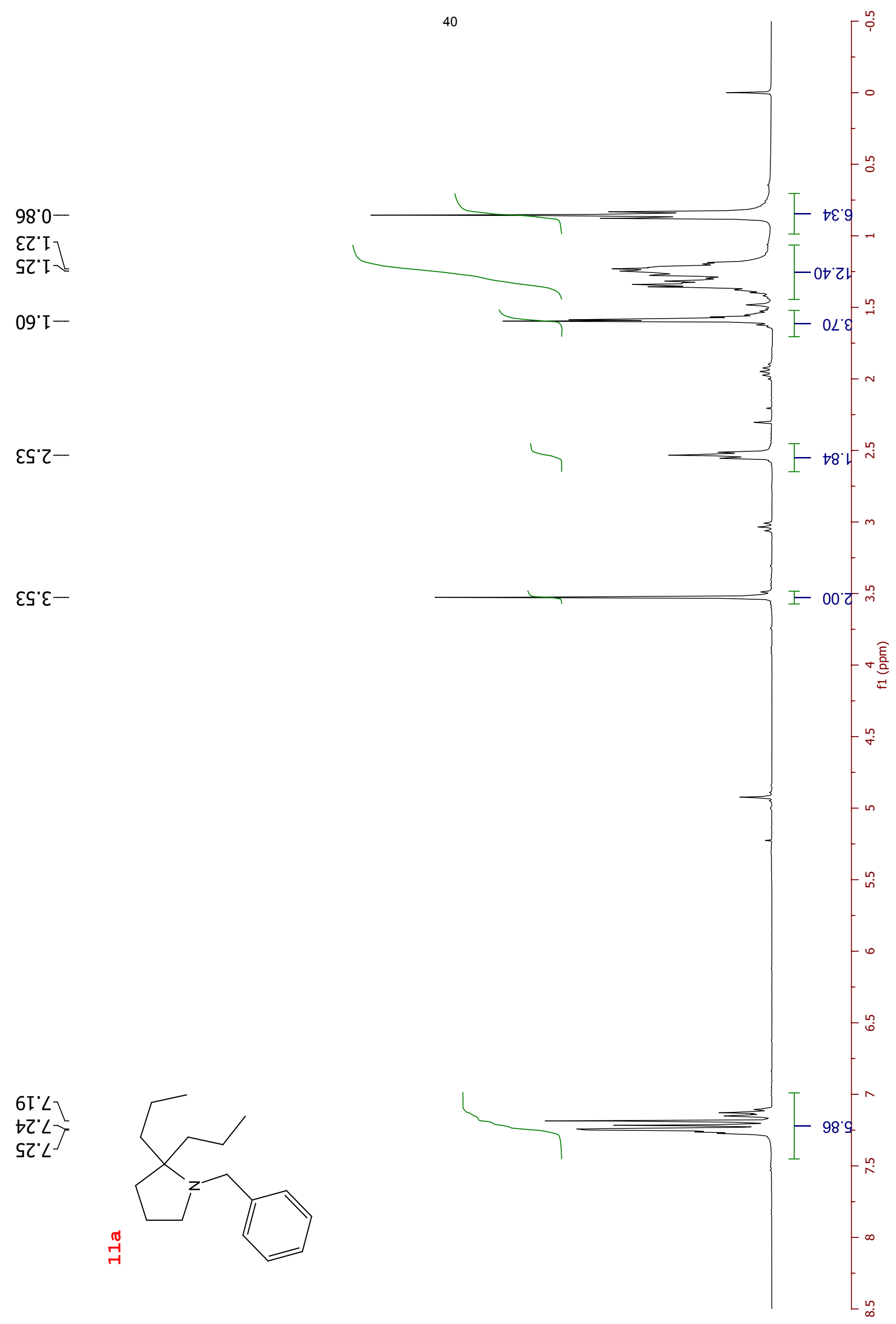




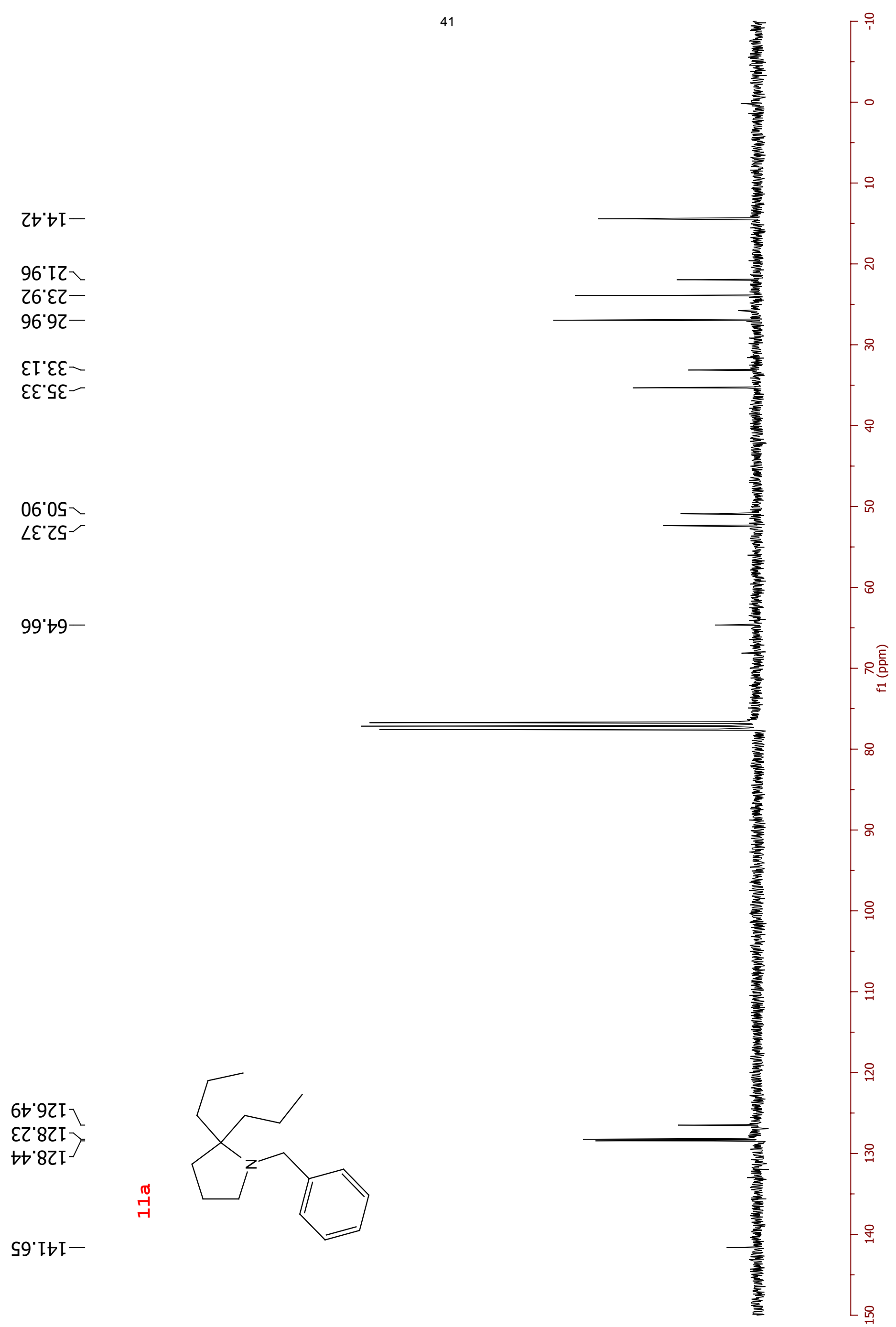




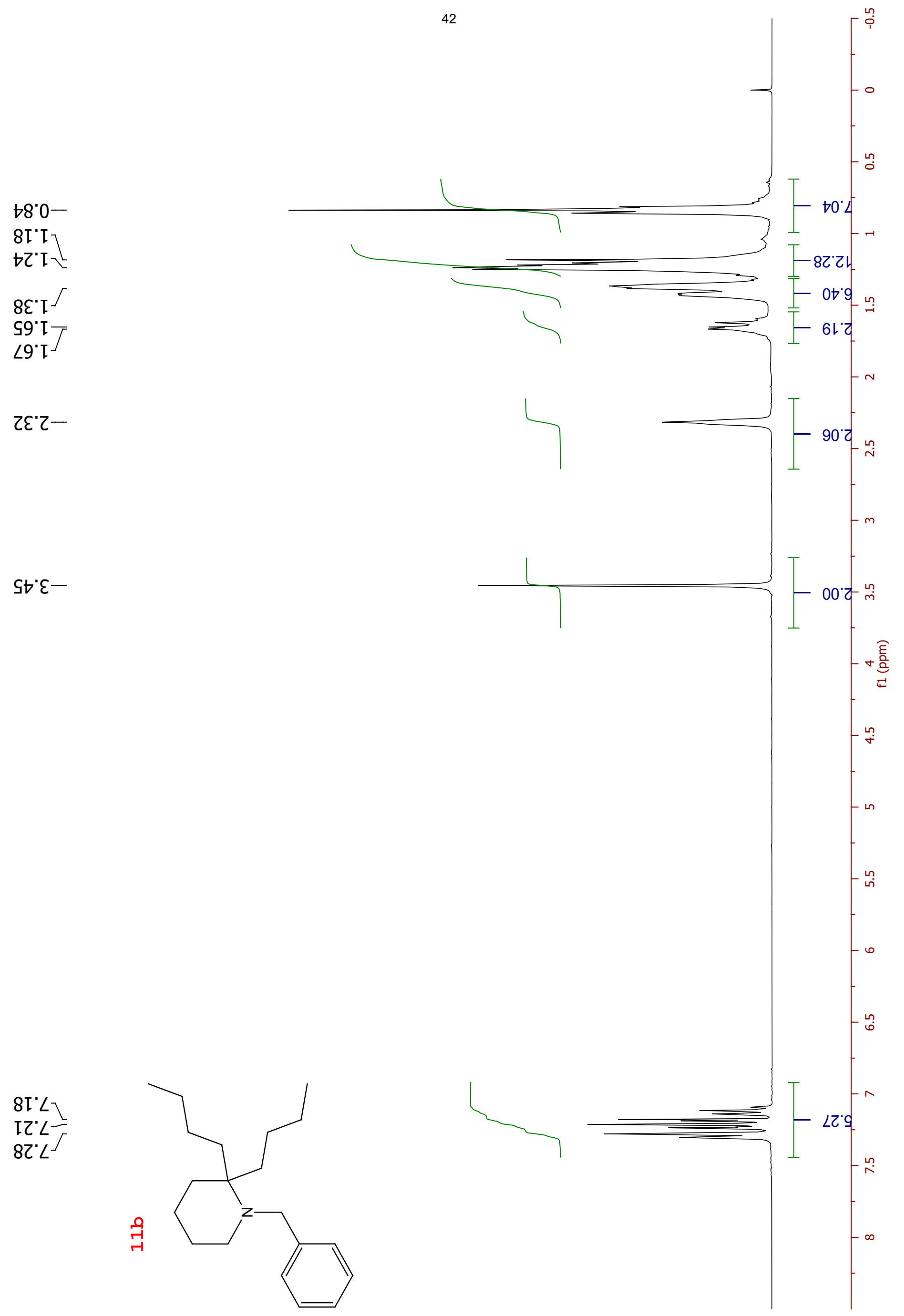


St' $\varepsilon \varepsilon$

86'St-

$26{ }^{\circ} 25-$

$S 9^{\circ} \angle S-$
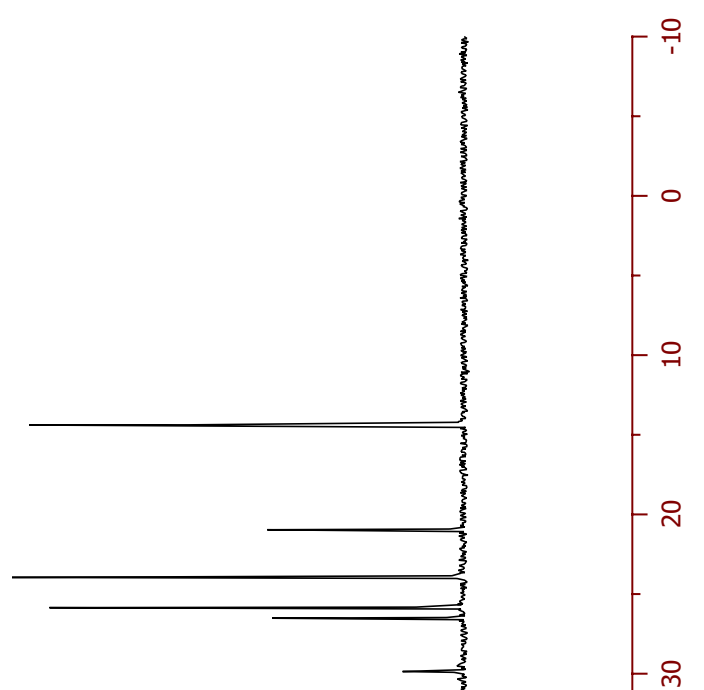

\section{$S \angle S$}

乙ย'9ZI -

हZ'8ZI-

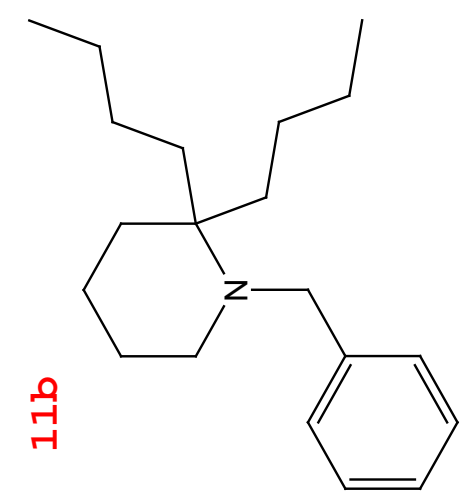

$6 L^{\prime}$ TtI-

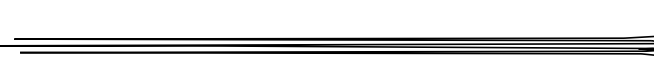


$000^{\circ}-$

$28^{\circ} 0$

$580^{\circ}$

$\angle 8^{\circ} O^{-}$

ZI'I-

I 2 ' -5

9Z' I

โE' I]

$\varepsilon b^{\prime} \cdot$

6S' [

$t S^{\prime} \mathrm{Z}$

95' $2 \frac{1}{5}$

$\downarrow I^{\prime} \varepsilon-$

$99^{\circ} \varepsilon-$
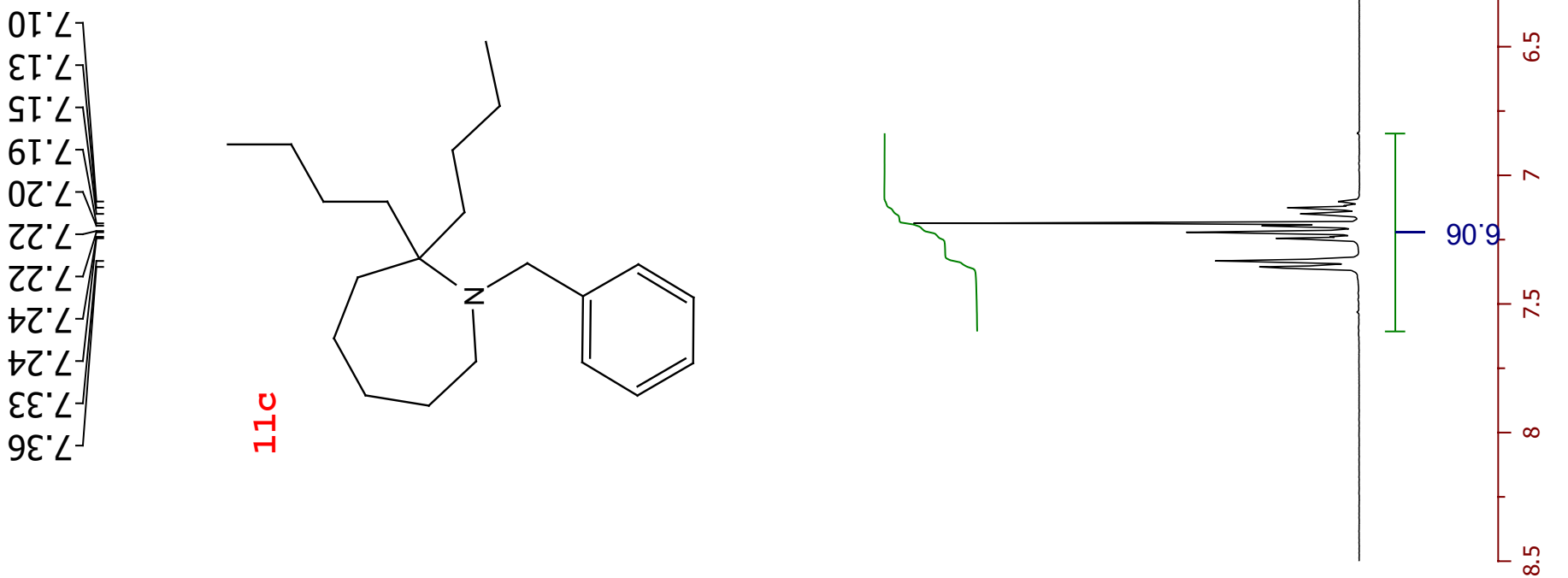


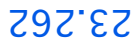

ll l"tट

$222 . \angle 2$

$898^{\circ} 62$

ट२2"0ह

Z9L'L

$\nabla \nabla \tau^{\circ} \angle \varepsilon$

$882^{\circ} 8 \varepsilon$

sEt $8 t$

0tヤ゙ts

192.09

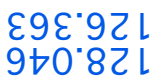

† 9 . 821

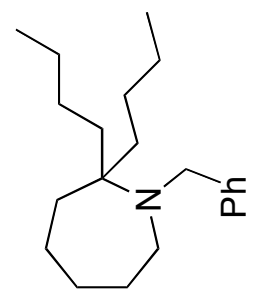

080"عt L

$\underset{r}{-}$

- ㄱ

이

아

은

요

웅

$\infty$

우

음

음

$\stackrel{ }{-}$

음

옴

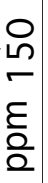


โ6' 2

$56^{\prime} 27$

ธด $0^{\circ} \varepsilon$

$\angle 0^{\circ} \varepsilon-$

$9{ }^{\prime} \varepsilon-$

20'৮一

乙ع'॰-

00 's-

tย's-

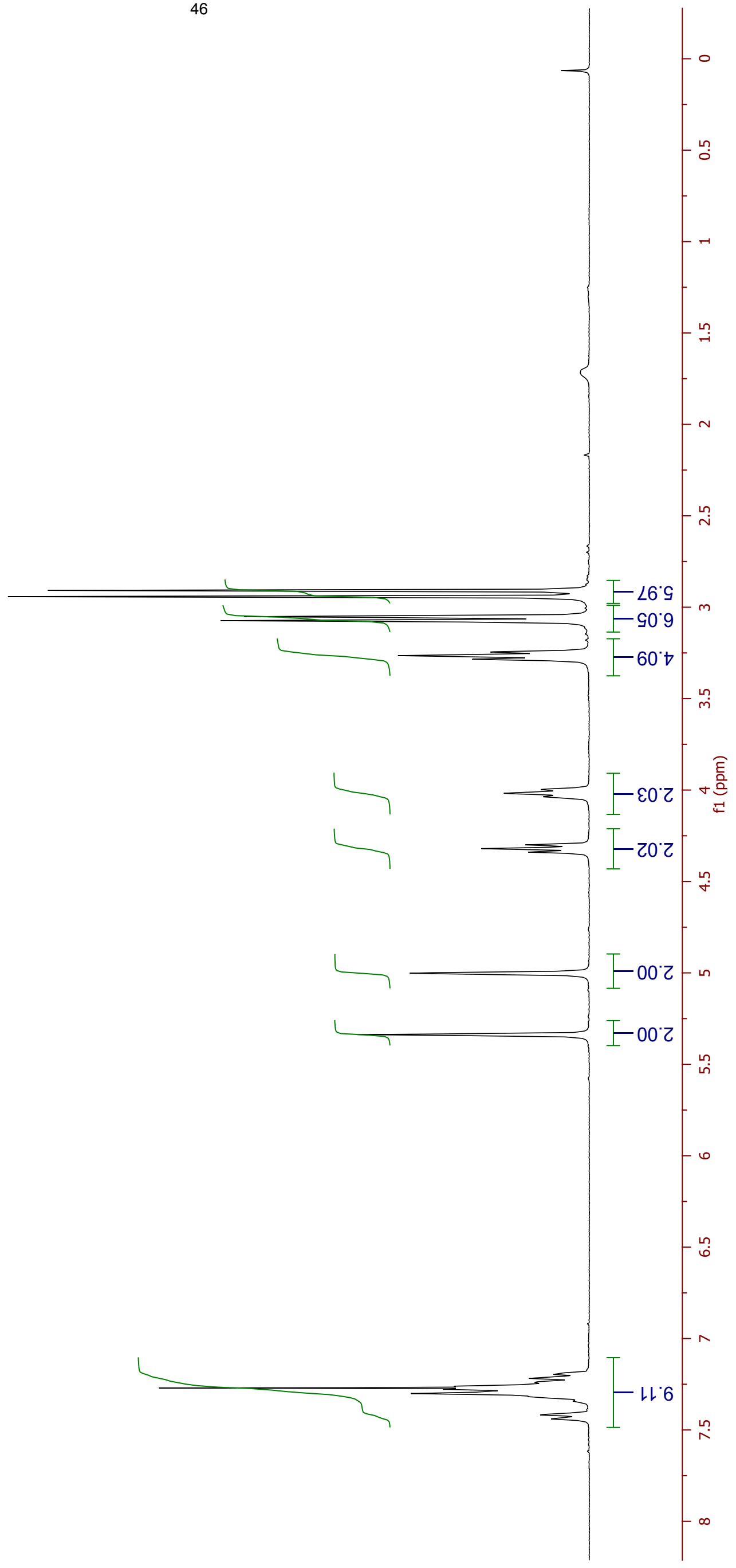


$9 s^{\prime} b 2$
$899^{\circ}+{ }^{\prime}$

$26^{\circ} \angle 2>$

It $82^{-1}$

†0'2S-

OI'ES-

$6 s^{\prime} t 5-$

ZI'9S-

S9.9ZI -

IZ'LZI]

$9 b^{\circ} \angle Z I$

$\left.0 L^{\circ} \angle Z T\right]$

$6 \angle \angle Z T$ E

I0.8ZI

$0 t^{\circ} 8 \mathrm{II}^{-}$

6L'8ZI

†6.8ZI

โ8'દยโ \

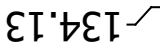
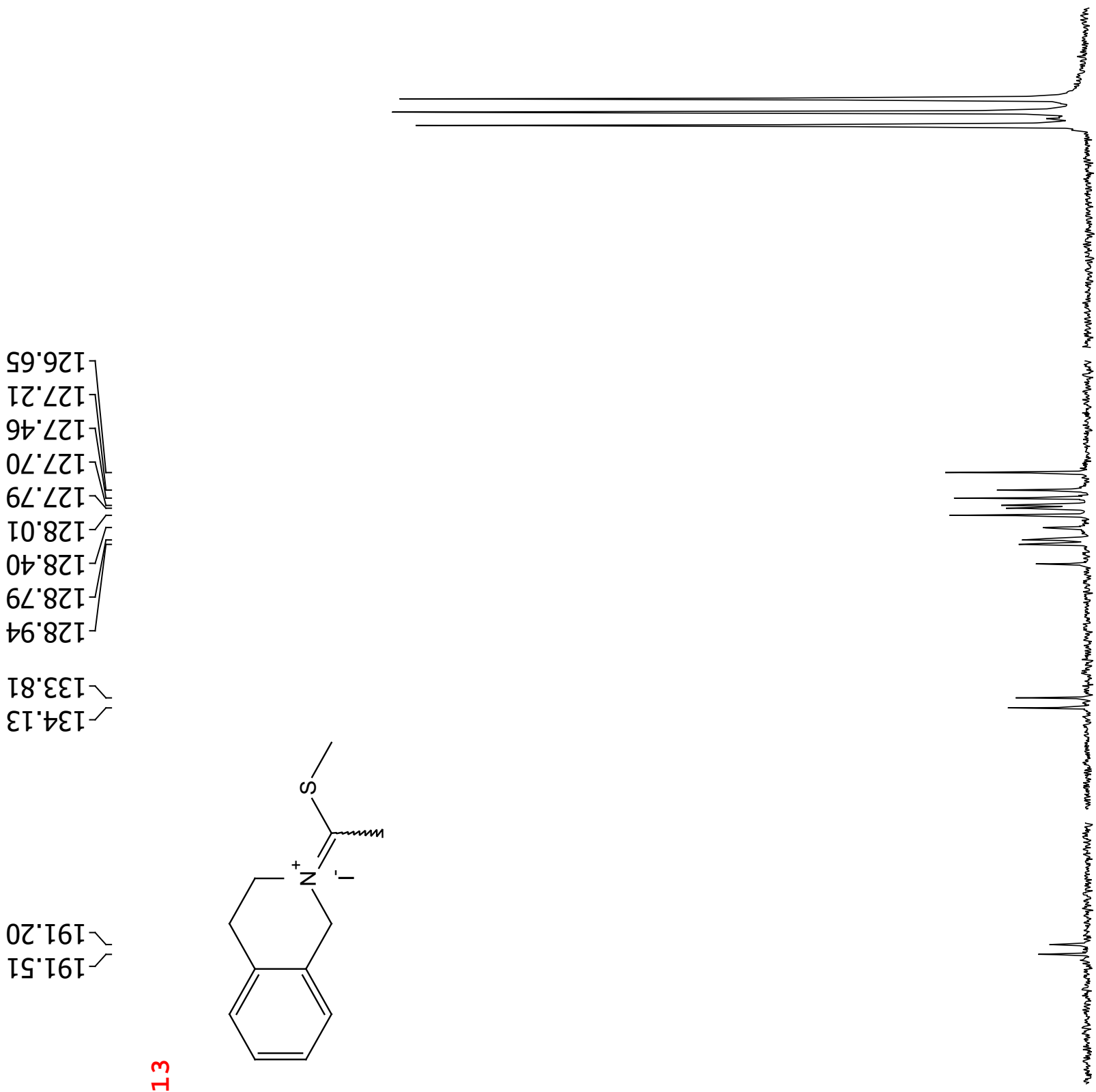
[8' 2

ย8 2

$68^{\prime} \tau^{\top}$

$08^{\circ} \varepsilon-$

OI'L-

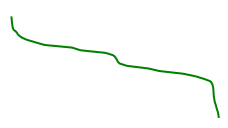

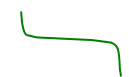

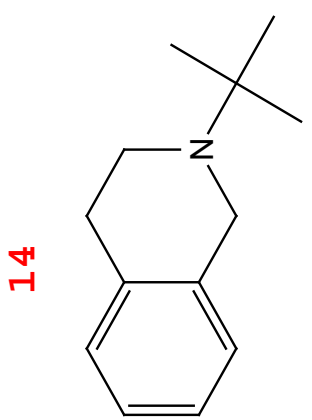

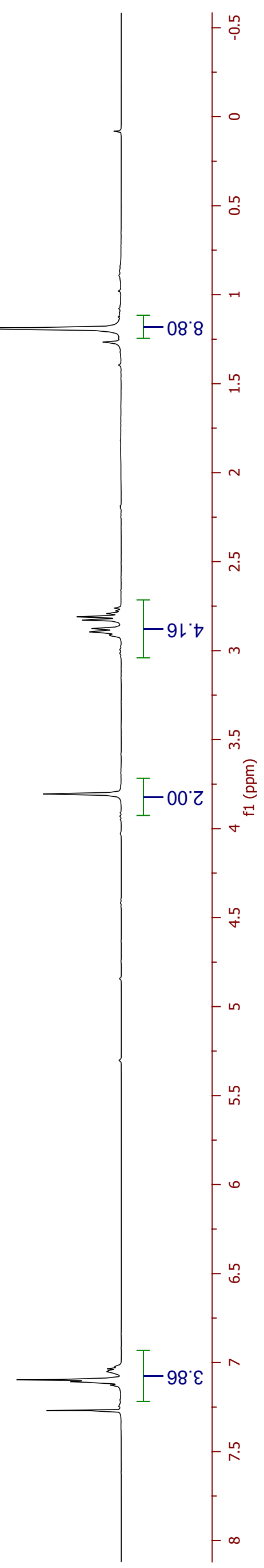


เ69.0E

৪๕乙゙ヤ

$\varepsilon \angle L^{\circ} 6 t$

s\&O"ts
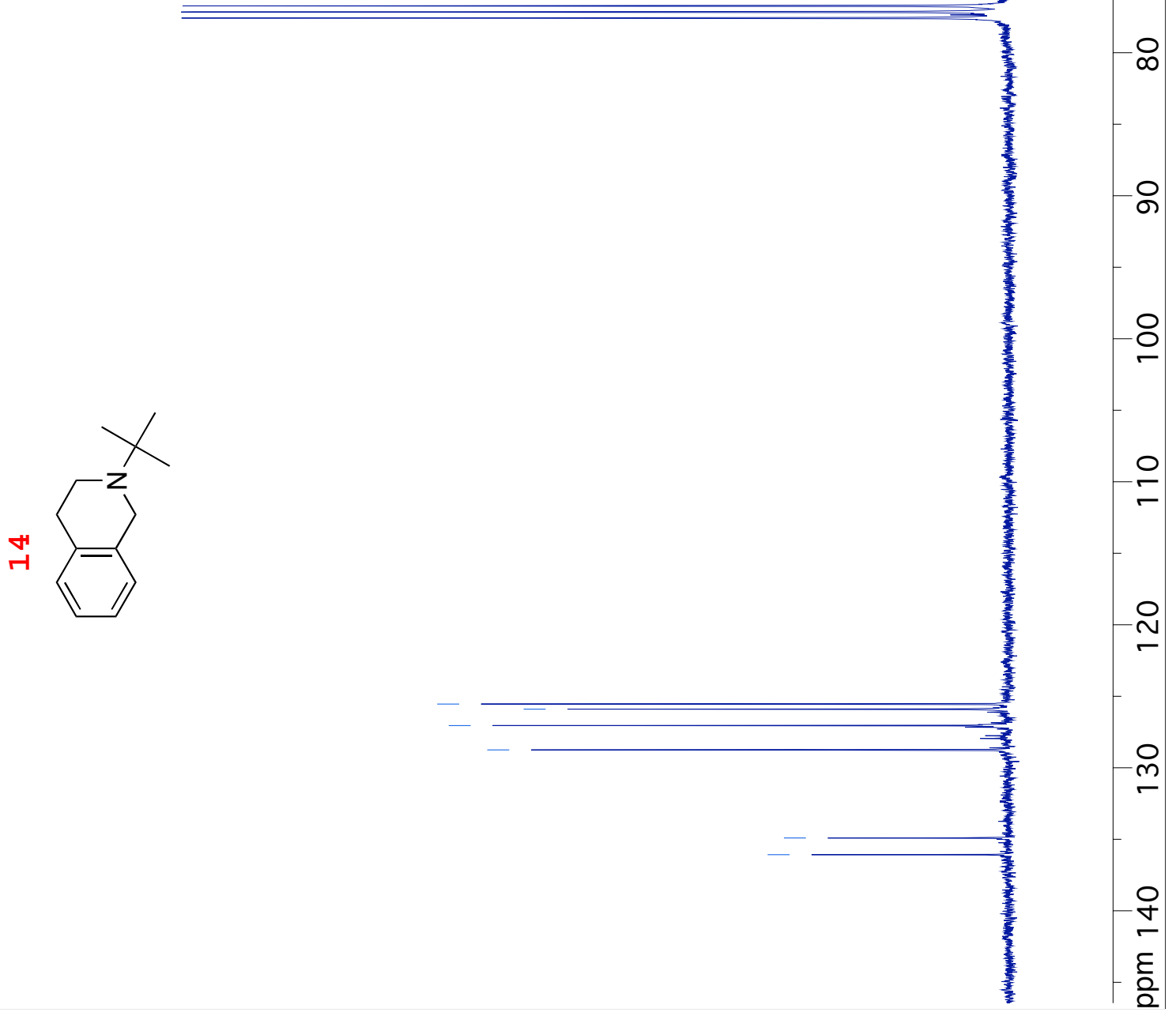